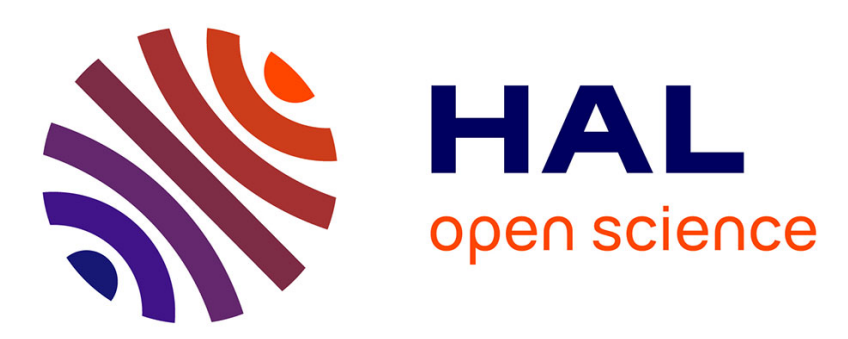

\title{
Cell-Kinetics Based Calibration of a Multiscale Model of Structured Cell Populations in Ovarian Follicles
}

Benjamin Aymard, Frédérique Clément, Danielle Monniaux, Marie Postel

\section{To cite this version:}

Benjamin Aymard, Frédérique Clément, Danielle Monniaux, Marie Postel. Cell-Kinetics Based Calibration of a Multiscale Model of Structured Cell Populations in Ovarian Follicles. SIAM Journal on Applied Mathematics, 2016, 76 (4), pp.1471-1491. 10.1137/15M1030327 . hal-01186381v2

\section{HAL Id: hal-01186381 \\ https://hal.science/hal-01186381v2}

Submitted on 31 Aug 2016

HAL is a multi-disciplinary open access archive for the deposit and dissemination of scientific research documents, whether they are published or not. The documents may come from teaching and research institutions in France or abroad, or from public or private research centers.
L'archive ouverte pluridisciplinaire HAL, est destinée au dépôt et à la diffusion de documents scientifiques de niveau recherche, publiés ou non, émanant des établissements d'enseignement et de recherche français ou étrangers, des laboratoires publics ou privés. 


\title{
CELL-KINETICS BASED CALIBRATION OF A MULTISCALE MODEL OF STRUCTURED CELL POPULATIONS IN OVARIAN FOLLICLES
}

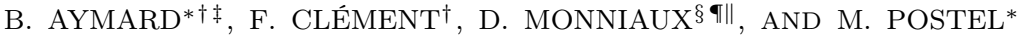

\begin{abstract}
In this paper, we present a strategy for tuning the parameters of a multiscale model of structured cell populations in which physiological mechanisms are embedded into the cell scale. This strategy allows one to cope with the technical difficulties raised by such models, that arise from their anchorage in cell biology concepts: localized mitosis, progression within and out of the cell cycle driven by time- and possibly unknown-dependent, and nonsmooth velocity coefficients. We compute different mesoscopic and macroscopic quantities from the microscopic unknowns (cell densities) and relate them to experimental cell kinetic indexes. We study the expression of reaching times corresponding to characteristic cellular transitions in a particle-like reduction of the original model. We make use of this framework to obtain an appropriate initial guess for the parameters and then perform a sequence of optimization steps subject to quantitative specifications. We finally illustrate realistic simulations of the cell populations in cohorts of interacting ovarian follicles.
\end{abstract}

Key words. transport equations, parameter calibration, structured cell populations, cell kinetics

AMS subject classifications. 92B05,35Q92

Introduction. In this paper, we deal with the question of the numerical calibration of an existing multiscale model of cell-structured populations in the physiological context of ovulation. This model was formulated as a system of weakly coupled, non conservative transport equations with controlled velocities and sink terms, where the unknowns are the cell densities in each follicle [9, 8]. A number of theoretical studies have established the well-posedness of the model [19], examined optimal control problems related to the ovulatory trajectories in the framework of hybrid optimal control theory [6], and studied the reachability of final states corresponding to either ovulatory or atretic cases in the framework of backwards reachable sets [8]. Implementation of the model in an efficient and reliable computing environment has involved the design of a finite-volume scheme dealing with the discontinuous coefficients [3], embedding this scheme within a dedicated adaptive mesh based on a multi-resolution approach [4], and implementing it on parallel architecture [2]. This has left the question of model calibration to biological specifications to be resolved.

We have to face a generic, yet unsolved issue in parameter fitting for physiologicallyoriented multiscale mathematical models: although mechanistic knowledge in molecular and cell biology is available on the lower scales, quantitative experimental data are rather available on the higher scales. In our case, the question is how to infer the parameters entering the microscopic functions (on the level of the follicular cells) from mesoscopic (on the level of the individual follicles, i.e. the number of follicular cells) or macroscopic (on the level of the populations of follicles) information. In addition, even on the macroscopic level, data remain rather scarce and are rarely obtained directly as

* Sorbonne Universités, UPMC Univ Paris 06, Laboratoire Jacques-Louis Lions, UMR CNRS 7598, F-75005, Paris, France

${ }^{\dagger}$ INRIA Paris Research Center, Mycenae Project-Team, 2 rue Simone Iff, CS 42112, 75589 Paris Cedex 12 Cedex - France

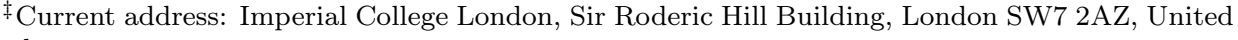
Kingdom

§INRA, UMR85 Physiologie de la Reproduction et des Comportements, F-37380 Nouzilly, France

I CNRS, UMR7247, F-37380 Nouzilly, France

"Université François Rabelais de Tours, F-37041 Tours, France; IFCE, F-37380 Nouzilly, France 
a function of time (but more often as relationships between different variables). Altogether, these difficulties preclude the exclusive use of standard optimization methods to obtain the parameter values. We have thus calibrated the model by combining a priori information on the parameters, deduced from biological knowledge or mathematical properties of the model functions, a posteriori diagnostic on some model outputs, and optimization of the fitting with respect to a training dataset. The resolution of this additional question is needed to fully exploit the model potential in terms of biological interpretation. Due to the anchorage of the model formulation within the concepts of cell biology, we also believe that our calibration strategy has a generic interest and could be applied in its principles to other biological situations. Indeed, even though it is an original character of the model to take into account a localized mitosis instead of a distributed growth rate -instead of a source term in the right-hand side as is almost systematically done for renewal equations [16]-, it is also one of its most generic trait since it is directly related to basic principles of cell biology. This trait is furthermore accompanied in our framework by time-dependent phase durations subject to the control of extracellular signals, and combined with cell death and differentiation in a 2D framework. On a formal ground, some authors have already proposed to associate the mitosis event with the reaching of a threshold on a structuring variable $[17,5]$ in a $1 \mathrm{D}$ framework. Yet, to our knowledge, they have not coped with the numerical problems generated by mitosis-induced discontinuity.

The paper is organized as follows. In section 1, we first recall the formulation of the multiscale model as weakly-coupled, non conservative transport equations, with space-dependent velocities and explicit accounting for the mitosis event and then we introduce macroscopic quantities associated with the microscopic unknowns (cell densities). Thereafter we present novel results. In section 2, we take advantage of the realistic (localized) modeling of mitosis to derive a cell kinetics marker (the mitosis index IM) and to describe the age distribution of cells along the cell cycle in dynamical frameworks of increasing complexity, paying special attention to the impact of the contrast in the velocities. In section 3, we introduce a simplified framework with piecewise constant velocities, that allows us to compute rigorously the reaching times of some characteristic transition zones in the computation domain. In section 4, we explain how to tune the parameters of the original model, first in the case of an ovulatory trajectory, and then in a situation of selection amongst a cohort of growing follicles. The final section is devoted to a discussion. The paper is accompanied by supplemental materials dealing with the numerical computation and simulation of cell kinetics indexes (Appendix A), the derivation of a priori constraints on the velocity parameters (Appendix B), and specific contextual elements for the application to ovarian follicles (Appendix C), including the design of specifications on the model outputs as well as the detailed sequence of the calibration steps reviewed in section 4 . The numerical results are substantiated by companion movies.

\section{Main features of a controlled multiscale model for structured cell populations in ovarian follicles.}

1.1. Model formulation. In this section, we recall in a compact way the formulation of the multiscale model representing the terminal development of a cohort of ovarian follicles and its control by pituitary hormone FSH (follicle-stimulating hormone). A more detailed formulation is provided in the beginning of Appendix B; we refer the interested reader to more biologically-oriented works $[9,7]$ for the introduction of the biological bases underlying the model formulation. We consider a cohort of $F$ interacting follicles. The cell density $\left(\phi_{f}(t, x, y)\right)_{f=1, \ldots, F}$ within each follicle satisfies 
the following system of equations, for $f=1, \ldots, F$ :

$$
\frac{\partial \phi_{f}}{\partial t}+\frac{\partial\left(g_{f}\left(x, y, u_{f}(t)\right) \phi_{f}\right)}{\partial x}+\frac{\partial\left(h_{f}\left(x, y, u_{f}(t)\right) \phi_{f}\right)}{\partial y}=-\Lambda(x, y, U(t)) \phi_{f},
$$

set in the computing domain $\Omega$ in the $(x, y)$ plane,

$$
\Omega=\left\{(x, y), 0<x<N_{c} \times D_{c}, 0<y<y_{\max }\right\}
$$

where $N_{c}$ is the number of cell cycles and $D_{c}$ is the duration of one cycle, and $y_{\max }$ a supremum of the maximum maturity that can be reached by the density. The $F$ equations are linked together through the control variables $u_{f}(t)$ and $U(t)$, that appear as arguments in the aging $g_{f}(x, y, u)$ and maturation $h_{f}(x, y, u)$ velocity, and also in the sink term $\Lambda(x, y, U)$. Both control variables are defined from the first maturity moment of the densities.

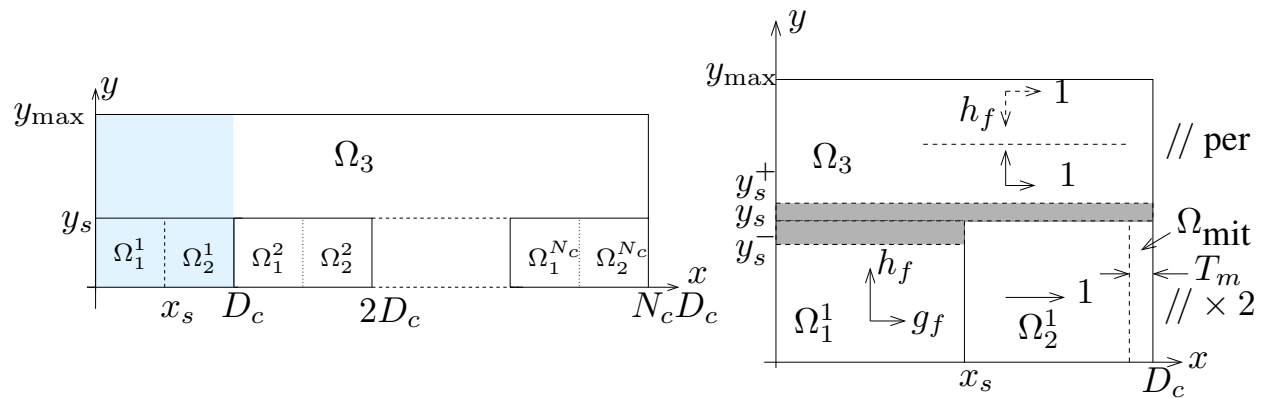

Figure 1.1. Left panel: computational domain with the subsequent cell cycles $\Omega_{1}^{p} \cup \Omega_{2}^{p}, p=$ $1, \ldots, N_{c}$ of length $D_{c} . \Omega_{1}^{p}$ corresponds to phase $G 1$, while $\Omega_{2}^{p}$ aggregates phases $S, G 2$ and $M$ of the pth cell cycle. Phase $\Omega_{3}$ corresponds to the zone of differentiation, which cells can enter from any $\Omega_{1}^{p}$ phase. Right panel: single cell cycle, with the different phases $G 1\left(\Omega_{1}^{1}\right), S M\left(\Omega_{2}^{1}\right)$ and $D\left(\Omega_{3}\right)$. The part of phase SM corresponding to the mitosis process $\left(\Omega_{m i t}\right)$ is delimited with a dashed line. The zone where cells are sensitive to apoptosis is highlighted in grey. The direction of the aging and maturation velocities are sketched with arrows. Periodic boundary conditions (// per) based on flux continuity are applied on the outer boundaries, with the mitosis-induced density doubling $(/ / \times 2)$ on the vertical boundary at $x=D_{c}$ for $y<y_{s}$.

As represented in the left panel of Figure 1.1, the domain $\Omega$ is divided in $2 N_{c}+1$ zones: $\Omega_{1}^{p}, \Omega_{2}^{p}$, for $p=1, \ldots, N_{c}$ and $\Omega_{3}$, corresponding to different cell states and hence different definition of the velocities and sink terms. Phase $\Omega_{1}^{p}$ corresponds to the G1 phase of the cell cycle. Phase $\Omega_{2}^{p}$ aggregates the three latest phases (S, G2, $\mathrm{M}$ ) of the $p^{\text {th }}$ cell cycle. Phase $\Omega_{3}$ corresponds to a differentiated state, where cells have exited the cell cycle and undergo terminal differentiation.

$$
\begin{cases}\Omega_{1}^{p}=\{(x, y) \in \Omega, & \left.p D_{c}<x<p D_{c}+x_{s}, 0<y<y_{s}\right\}, p=0, \ldots, N_{c}-1, \\ \Omega_{2}^{p}=\{(x, y) \in \Omega, & \left.p D_{c}+x_{s}<x<(p+1) D_{c}, 0<y<y_{s}\right\}, p=0, \ldots, N_{c}-1, \\ \Omega_{3}=\{(x, y) \in \Omega, & \left.y_{s}<y\right\}, \Theta_{1}=\cup_{p=1}^{N_{c}} \Omega_{1}^{p}, \Theta_{2}=\cup_{p=1}^{N_{c}} \Omega_{2}^{p} .\end{cases}
$$

Each cell cycle consists of the $\Omega_{1}^{p} \cup \Omega_{2}^{p}$ subdomain and $\Theta_{i}$ for $i=1,2$ denotes the disconnected union of the $N_{c}$ corresponding phases $\Omega_{p}^{i}$, for $p=1, \ldots, N_{c}$. In $\Omega_{1}$, both $g_{f}$ and $h_{f}$ are positive functions, monotonically increasing with $u_{f}$ (see details in Eq. B.1-B.2). In $\Omega_{2}$, the dynamics correspond to a simple horizontal transport, where age evolves as time $\left(g_{f}=1\right.$ and $\left.h_{f}=0\right)$. Due to its quadratic expression in $y, h_{f}$ changes 
sign within $\Omega_{3}$.

The support of the initial condition $\phi_{f}(0, x, y)=\phi_{0 f}(x, y)$ has to satisfy

$$
\text { Supp. } \left.\phi_{0 f}=\right] 0,1\left[\times\left[\mu_{1}, \mu_{2}\right] \text { with } 0<\mu_{1}<\mu_{2}<y_{s}\right.
$$

such as in (2.10) for instance. The precise definition of the required transmission conditions along the successive cell cycles of the domain has been formulated in [19]. For each cycle $p=1, \ldots, N_{c}$,

- the flux on the $x$-axis is continuous on the interface between $\Omega_{1}^{p}$ and $\Omega_{2}^{p}$, for all $p=1, \ldots, N_{c}$

$$
\phi_{f}\left(t, x^{+}, y\right)=g_{f} \phi_{f}\left(t, x^{-}, y\right), \quad x=p D_{c}-x_{s}, \quad 0<y<y_{s} .
$$

- The flux is doubling on the interface between $\Omega_{2}^{p}$ and $\Omega_{1}^{p+1}$, which accounts for the birth of two daughter cells from one mother cell (mitosis) at the end of each cell cycle, for all $p=1, \ldots, N_{c}-1$

$$
g_{f} \phi_{f}\left(t, x^{+}, y\right)=2 \phi_{f}\left(t, x^{-}, y\right), \quad x=p D_{c}, \quad 0<y<y_{s}
$$

- A homogeneous Dirichlet condition holds to the north of the interface between $\Omega_{2}^{p}$ and $\Omega_{3}$

$$
\phi_{f}\left(t, x, y_{s}^{+}\right)=0, \quad p D_{c}-x_{s}<x<p D_{c} .
$$

In the age variable $x$, the velocity is always strictly positive, therefore the number of cell cycles $N_{c}$ can be tuned so as to cover, in as much as possible, the expected time horizon of the simulation, so that no cell may reach the right outer boundary before the end of the simulation. However, to guarantee that the numerical problem be well defined in all cases, we also set boundary conditions on the vertical outer boundaries of the domain:

$$
g_{f} \phi_{f}\left(t, 0^{+}, y\right)=2 \phi_{f}\left(t, N_{c} D_{c}^{-}, y\right), \quad x=p D_{c}, \quad 0<y<y_{s} .
$$

Combined with a choice of a sufficiently large $N_{c}$, condition (1.6) still allows one to follow the motion and spreading of the density over successive cycles on the unrolled domain (left panel Figure 1.1) for a while. Once the condition becomes active, the density reaching the right end on the domain folds itself and starts again from the left end of the domain. In that case, one can continue to track the cell ages, modulo $N_{c} D_{c}$. Alternatively, for the sake of computational purposes, condition (1.6) can be combined with a single cell cycle domain (right panel Figure 1.1). In that case, the dynamics remain globally unchanged, and the cell age still remains informative on the cell progression along the cycle, but it is no more possible to follow the absolute cell age, since it is reset at the beginning of the cycle, neither the generation lineage of cells.

1.2. Macroscopic outputs associated with the cell densities. The model outputs can span several space scales, from the microscopic scale of the local cell density to the macroscopic scale of moments computed over the whole domain $\Omega$. The first order moments in maturity are embedded in the model formulation since they are the basis for the coupling between the PDE corresponding to each follicle (see details in B.6 and B.7). We distinguish the individual follicular maturity $m_{f}^{1}(t)$ 
and the global ovarian maturity $M(t)$ :

$$
\begin{aligned}
m_{f}^{1}(t) & =\int_{0}^{1} \int_{0}^{N_{c} D_{c}} y \phi_{f}(t, x, y) \mathrm{d} x \mathrm{~d} y, \\
M(t) & =\sum_{f=1}^{F} m_{f}^{1}(t) .
\end{aligned}
$$

Other macroscopic quantities are useful from the calibration viewpoint since they are directly related to observable variables in the field of experimental cell kinetics. The total cell number, that can be assessed from sampled countings, is simply the zero-order moment of the density in both age and maturity $m_{f}^{0}(t)$

$$
m_{f}^{0}(t)=\iint_{\Omega} \phi_{f}(t, x, y) \mathrm{d} x \mathrm{~d} y .
$$

Amongst these cells, the number of cells lying within the proliferating part of the domain, $\Omega_{1}^{p}, \Omega_{2}^{p}$, for $p=1, \ldots, N_{c}$, that can also be simply denoted as $\Omega \backslash \Omega_{3}$, allows us to introduce the growth fraction $G F$, which is the proportion of proliferating cells amongst the whole population. The growth fraction can be assessed by means of cell labeling protocols that detect the passages of cells throughout the S phase of DNA replication [13].

$$
G F(t)=\frac{\iint_{\Omega \backslash \Omega_{3}} \phi_{f}(t, x, y) \mathrm{d} x \mathrm{~d} y}{\iint_{\Omega} \phi_{f}(t, x, y) \mathrm{d} x \mathrm{~d} y} .
$$

1.3. Dealing with the coupling terms. The coupling of equations through moments adds to the difficulty of calibrating the parameters. In the next sections, we will thus consider alternatively different situations

- the uncoupled case with no cell death, considering a single best-case, ovulatory trajectory that can be studied in the absence of cell death, with an open-loop control term as that defined in (2.9). This situation captures the switch from proliferation to differentiation to follow the increase and subsequent stabilization of the total cell number, as the result of a speed composition problem ruling the final total cell number and the time when all cells have exited the cell cycle $\left(T_{e}\right)$.

- the auto-coupled case, where a single ovulatory follicle is considered, but the weak nonlinearity of the model is preserved (moment-dependent expressions of the velocities and sink term). In this situation, the apoptotic process interacts with proliferation and cell cycle exit and the increase in follicular maturity has to be balanced in a timely way to avoid premature cell loss.

- the original case considering a cohort of $F$ interacting follicles, which embeds all the model specificities and where any follicle trajectory is impacted by the pressure exerted by the rest of the cohort.

\section{Computation of cell-kinetics indexes from the model outputs.}

2.1. Definition of the mitotic index and age distribution within the cell cycle. To follow the progression of cells along the cell cycle in more detail, we can 
also compute the distribution in age within the proliferative part of the domain $\psi(t, x)$ (we drop the $f$ index to simplify notations):

$$
\psi(t, x)=\int_{\Omega \backslash \Omega_{3}} \phi_{f}(t, x, y) \mathrm{d} y .
$$

This distribution notably gives information about the degree of between-cells synchronization. For instance, a fully desynchronized cell population with $\mathrm{GF}=1$ will be distributed according to an exponential distribution with twice as many "young" cells (that have just entered the G1 phase after mitosis) as "old" cells (that are about to divide at the end of the mitosis phase) [1].

From the age distribution, we can compute the mitotic index (MI), which assesses the proportion of cells undergoing mitosis. For the sake of concision, we will introduce the principles of MI computation from the age distribution $\psi(x, t)$ and express the MI as the proportion of mitotic cells within the population of proliferating cells

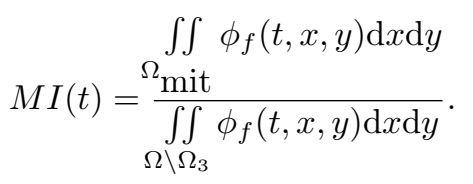

Expression with respect to the total cell population can be simply obtained by multiplying the MI value by the growth fraction defined in (1.9).

In the model, the mitosis phase is embedded within the SM phase, but we can nevertheless delimit a subpart of the SM phase corresponding specifically to mitosis $\left(\Omega_{\text {mit }}\right.$ in right panel of Figure 1.1). Since the aging velocity is constant in phase SM, and equals 1 (the cell age evolves as time), the relative length of the $M$ phase subpart within the SM phase can be deduced from the relative duration of mitosis $T_{m}$ (on the order of $30 \mathrm{~min}$ ) with respect to the whole duration of the SM phase $T_{S M}$ (on the order of 8 hours [11]). From the numerical viewpoint, the assessment of MI, original in this paper, requires to identify the meshes of the computation domain whose intersection with $\Omega_{m i t}=\left\{(x, y), p-T_{m}<x<p\right.$, for $\left.p=1, \ldots N_{c}, 0<y<y_{s}\right\}$ is non void (see right panel Figure 1.1). It can happen that such meshes overlap only partially with $\Omega_{m i t}$. In such a case, the contribution of a mesh to the numerator of the index is proportional to the mesh area included within $\Omega_{m i t}$, and we can compute an approximation $M I^{n}$ of MI at any time step $t_{n}$ as:

$$
M I^{n}:=\frac{\sum_{\Omega_{i, j} \cap \Omega_{\text {mit }} \neq \emptyset}\left|\Omega_{i, j} \cap \Omega_{\text {mit }}\right| \phi_{i, j}^{n}}{\sum_{\Omega_{i, j} \subset \Omega \backslash \Omega_{3}}\left|\Omega_{i, j}\right| \phi_{i, j}^{n}} .
$$

2.2. Simple exponential cell dynamics. We introduce here the computation of the MI in the simplest case of a desynchronized and fully proliferating cell population, with a constant velocity $g$ of progression along the cell cycle, and without any cell cycle exit process such as differentiation or apoptosis. In the $2 \mathrm{D}$ framework described by the master equation (1.1), we thus remove the sink term, set the maturation function to a constant null value, and initialize the density uniformly in maturity over the whole first cell cycle. With these assumptions, the 2D framework, in which the maturity distribution is uniform in space and constant over time, amounts to a 1D framework,

$$
\partial_{t} \psi(x, t)+\partial_{x}(g \psi(x, t))=0
$$


but we can still follow the progression of the cell density on the 2D domain (rather than on a line), which makes the visualization easier. In this simple framework, we can derive directly the computation of the MI. Starting from an exponential initial condition $\psi_{0}(x)=C e^{-k x}, C$ and $k$ are chosen such that $\psi_{0}\left(0^{+}\right)=\psi_{0}\left(D_{c}^{-}\right)$and $\int_{0}^{D_{c}} \psi_{0}(x) \mathrm{d} x=1$, which leads to $k=\frac{\ln 2}{D_{c}}$ and $C=\frac{2 \ln 2}{D_{c}}$.

The simplified transport equation (2.2) with periodically-doubling boundary conditions $\psi\left(t, 0^{+}\right)=2 \psi\left(t, D_{c}^{-}\right)$provides the solution for the density at all time

$$
\psi(t, x)=C e^{-\ln 2 \frac{g t}{D_{c}}} e^{-k x}=2^{\frac{g t}{D_{c}}} \psi_{0}(x),
$$

which highlights the stationary nature of the age distribution. From this formula, we can recover the macroscopic outputs, defined earlier, which come into play in the definition of the MI. The total cell number at time $t$ is

$$
N(t)=\int_{0}^{D_{c}} \psi(t, x) d x=\int_{0}^{D_{c}} 2^{\frac{g t}{D_{c}}} \psi_{0}(x) d x=2^{\frac{g t}{D_{c}}} \frac{C}{k}\left(e^{-k D_{c}}-1\right)=2^{\frac{g t}{D_{c}}} .
$$

The number of cells within $\Omega_{m i t}$ at time $t$ is

$$
N_{m i t}(t)=\int_{D_{c}-T_{m}}^{D_{c}} \psi(t, x) d x=2^{\frac{g t}{D_{c}}}\left(e^{k T_{m}}-1\right)
$$

Therefore, for all $t, 0 \leq g t \leq 1$ the mitotic index (2.1) is constant and equal to

$$
M I(t)=e^{k T_{m}}-1=e^{\frac{\ln 2}{D_{c}} T_{m}}-1,
$$

which is a classical formula (see e.g. [20]). The $x, y$ functional domain can also be used to illustrate the progression from one cell cycle to the subsequent one; instead of a periodic domain in $x$ we unfold the subsequent cell cycles in order to keep track of the cell generation (see Figure A.1 in Appendix A.1 for a comparison of the domains). Redefining the initial condition as

$$
\psi_{0}(x)= \begin{cases}C e^{-k x} & \text { for } 0<x \leq D_{c} \\ 0 & \text { for } D_{c}<x\end{cases}
$$

the solution is transported with velocity $g$ and doubled when it crosses the mitosis interface $x=n D_{c}, n=1,2, \ldots$. A simple recursion leads to the general solution, for $t \geq 0, x \geq 0$

$$
\psi(t, x)= \begin{cases}2^{n} C e^{-k(x-g t)} & \text { for } n D_{c}<x<(n+1) D_{c} \text { and }\left(x-D_{c}\right) / g \leq t \leq x / g \\ 0 & \text { elsewhere }\end{cases}
$$

2.3. Phase dependent cell dynamics: impact on the progression of cells along the cell cycle. We now consider the case of a cell cycle with piecewise constant, phase-specific velocities: $g \neq 1$ in $G 1$ phase and $g=1$ in $S M$ phase. To take into account the velocity discontinuity on the interface between the $G 1$ and $S M$ phase, we introduce a discontinuous initial condition,

$$
\psi(0, x)=\psi_{0}(x)=\left\{\begin{array}{ll}
M_{0} C_{G 1} e^{-k x_{s}} & \text { for } 0<x \leq x_{s} \\
M_{0} C_{S M} e^{-k x_{s}} & \text { for } x_{s}<x<D_{c}
\end{array},\right.
$$


subject to the normalization condition

$$
\int_{0}^{D_{c}} \psi_{0}(x) \mathrm{d} x=M_{0}
$$

We impose conditions of flux continuity at $x=x_{s}$ and flux doubling on the periodic boundary $x=0$ and $x=D_{c}$

$$
g C_{G 1} e^{-k x_{s}}=C_{S M} e^{-k x_{s}} \quad g C_{G 1}=2 C_{S M} e^{-k D_{c}}
$$

from which we obtain

$$
k=\ln (2) / D_{c}, \quad C_{G 1}=\frac{2 \ln (2)}{D_{c}\left(2+2^{\frac{1-x_{s}}{D_{c}}}(g-1)-g\right)}, \quad C_{S M}=g C_{G 1}
$$

and, replacing in (2.6),

$$
\psi_{0}(x)=\left\{\begin{array}{ll}
M_{0} C_{G 1} 2^{-x / D_{c}} & \text { for } 0<x \leq x_{s} \\
M_{0} C_{S M} 2^{-x / D_{c}} & \text { for } x_{s}<x<D_{c}
\end{array} .\right.
$$

The solution to (2.2) is no longer explicit but can be derived recursively, as done in [4] and recalled in Appendix A.2. We have computed numerically the master equation from this recursiveness approach, with $v_{p}=1 / 2$ for odd $p$ indexes and $v_{p}=1$ for even $p$ indexes to mimic the alternation between $G 1$ and $S M$ phases. To simplify we set $x_{s}=1 / 2$ and $D_{c}=1$. The solution is displayed in Figure 2.1. The density is displayed at initial time (left panel) and after one cell cycle (center panel) with a color code highlighting the discontinuity at $x_{s}=0.5$. The right panel displays the time evolution of macroscopic outputs (total cell number and mitotic index) in the case when the solution is computed from the recursiveness formula (A.5) (green lines) or from the Finite Volume method (blue lines). The lines are almost superimposed, which highlights the very good accuracy of the Finite-Volume numerical approximation.
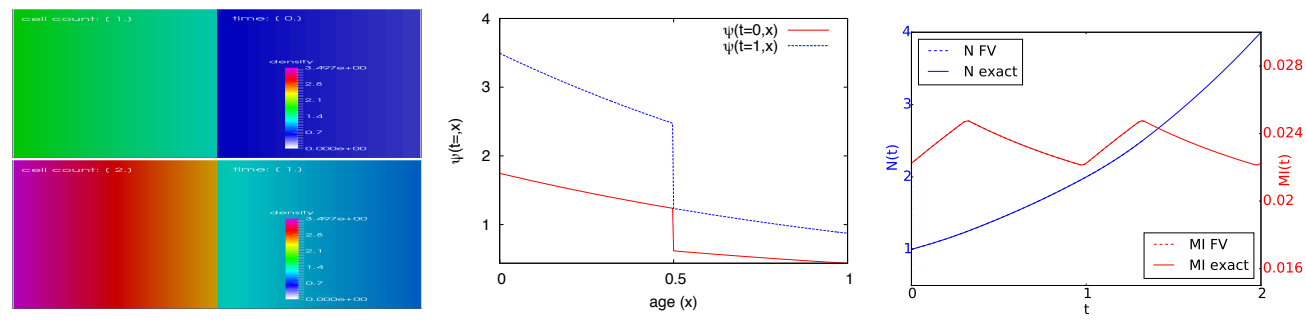

Figure 2.1. Left panel: snapshots of the cell density $\phi(t, x, y)$ over the $2 D$ domain (color-code panels) in the case of a fully proliferating, desynchronized cell population, with velocity contrast between the $G 1(g=0.5)$ and $S M(g=1)$ phases, at $t=0.0$ (top) and $t=1$ (bottom). Notice the discontinuity in the initial age distribution coming from (2.6). Center panel: age density $\psi(t, x)$ at $t=0$ and $t=1$. The shape of the density is periodically recovered after one cell cycle, and the cell number has doubled. Right panel: macroscopic outputs, total cell number (red) and mitotic index (blue). The dashed lines (computed from the Finite Volume method) and the solid lines computed from the recursiveness formula (A.5) are superimposed. 
2.4. Microscopic and macroscopic outputs in the case of cell cycle exit induced by differentiation and/or apoptosis. In our multiscale model, there is not only a contrast in the velocities according to the phases of the cell cycles, but also, due to the control terms, the velocities are time-varying. Moreover, cells are subject both to differentiation and apoptosis in phase G1, which affects the age distribution of cells within the cell cycle, hence the kinetics indexes such as MI and GF.

In this section, we design the simplest setup that can account for these facts and can help illustrate the changes observed in the model outputs, without coping yet with the full complexity of the model. To this end, we have to consider the genuine $2 \mathrm{D}$ character of the model, and introduce the time-varying and maturity-dependent expression for $h_{f}$, as defined in (B.2), and we need to deal with the sink term and the apoptosis rate formulated in (B.5), but we can bypass the closed-loop definition of $U(t)$ in (B.6), to impose instead a simple open-loop time pattern to $U(t)$. In addition, we forget about the distinction between $U(t)$ and the local control $u_{f}(t)$. More precisely, we consider the following piecewise constant function of time:

$$
u_{f}(t)=U(t)=U^{o l}:=\left\{\begin{array}{ll}
U_{\max }, & \text { for } t \leq T_{s} \\
U_{\min }, & \text { for }>T_{s}
\end{array}, \text { where } T_{s}\right. \text { is the switching time. }
$$

Also, in this setup, the parameter values are not intended to take realistic values (i.e. values that would lead to model outputs meeting the quantitative biological specifications), but they are chosen so that a complete cell cycle exit can occur rapidly and be observed as soon as after 2 cell cycles have elapsed. The aging velocity is kept unchanged with respect to the former subsections, with $g \neq 1$ in phase G1 and $g=1$ in SM. The initial condition (1.2) is now uniformly spread over the initial maturity range $\left[\mu_{1}, \mu_{2}\right]$

$$
\phi_{0}(x, y)=\frac{1}{\mu_{2}-\mu_{1}} \psi_{0}(x) \mathbb{1}_{\left[s_{1}, s_{2}\right]}(y)
$$

where $\psi_{0}$ is defined by $(2.8)$.

We comment in Appendix A.3 on a detailed simulation corresponding to this dynamical framework* and illustrating the impact of cell cycle exit and apoptosis on the model outputs on different scales and exhibiting the long time behavior of the solution theoretically studied in [14].

3. Characteristic times of the cell dynamics. We address here the problem of computing critical times corresponding to the sequential reaching of the different zones that structure the domain vertically, within a simplified dynamical framework consisting in a particle-like reduction of the original PDE model. The results of this section will be exploited as a central step in our calibration strategy (see section 4.1 completed by Appendix C.3.1) to obtain an appropriate initial guess of the whole parameter set despite little a priori information.

For the sake of convenience, we will design by " $\mathrm{P}+\mathrm{A}$ ", the zone where apoptosis is superimposed with proliferation, and " $\mathrm{D}+\mathrm{A}$ " that where it is superimposed with differentiation. We are specially interested in times $T_{P+A}$ of entry into the apoptosissensitive area, $T_{D+A}$ of exit from the apoptosis-sensitive area and $T_{e}$ of exit from the cell cycle. Between $T_{P+A}$ and $T_{e}$, cells are both still proliferating and possibly subject to apoptosis, while, between $T_{e}$ and $T_{D+A}$, cells have exited the cell cycle and

\footnotetext{
${ }^{*}$ An animation displaying the cell density evolution is also available in the supplementary material.
} 
proliferation cannot compensate even partially for possible cell loss through apoptosis. As we will see, these times do not only depend on the initial maturity, but also on the initial age for cells sharing the same maturity, as a consequence of the drift transport dynamics in phase SM (subpart $\Omega_{2}$ in the domain).

To make possible the direct computation of the cell travel times, we bypass the closedloop definition of $U(t)$ in (B.6), to impose instead a simple open-loop expression similar as the piecewise-constant pattern already introduced in (2.9)

$$
U(t)=U^{o l}:=\left\{\begin{array}{ll}
U_{\max }, & \text { for } t \leq T_{s 2} \\
U_{\min }, & \text { for }>T_{s 2}
\end{array} .\right.
$$

The time-evolution of the local control $u_{f}^{o l}(t)$ is defined within the $U_{\min }$ and $U_{\max }$ bounds of $U(t)$ as

$$
u_{f}^{o l}(t)=\left\{\begin{array}{l}
b_{1} U_{\max }, \quad \text { for } t \leq T_{s 1} \\
\beta_{2} U_{\max }, \quad \text { for } T_{s 1}<t \leq T_{s 2} \\
U_{\min }, \quad \text { for } t>T_{s 2}
\end{array},\right.
$$

where $b_{1}$ is the same parameter as in the $b_{f}$ function (see Eq. B.7), $\beta_{2}$ controls the height of the second plateau $\left(b_{1}<\beta_{2}\right), T_{s 1}$ and $T_{s 2}$ are switching times. This piecewise constant pattern amounts to an open-loop approximation of the pattern followed by $u_{f}(t)$ in the closed-loop situation, as illustrated in left panel of Figure 3.1.
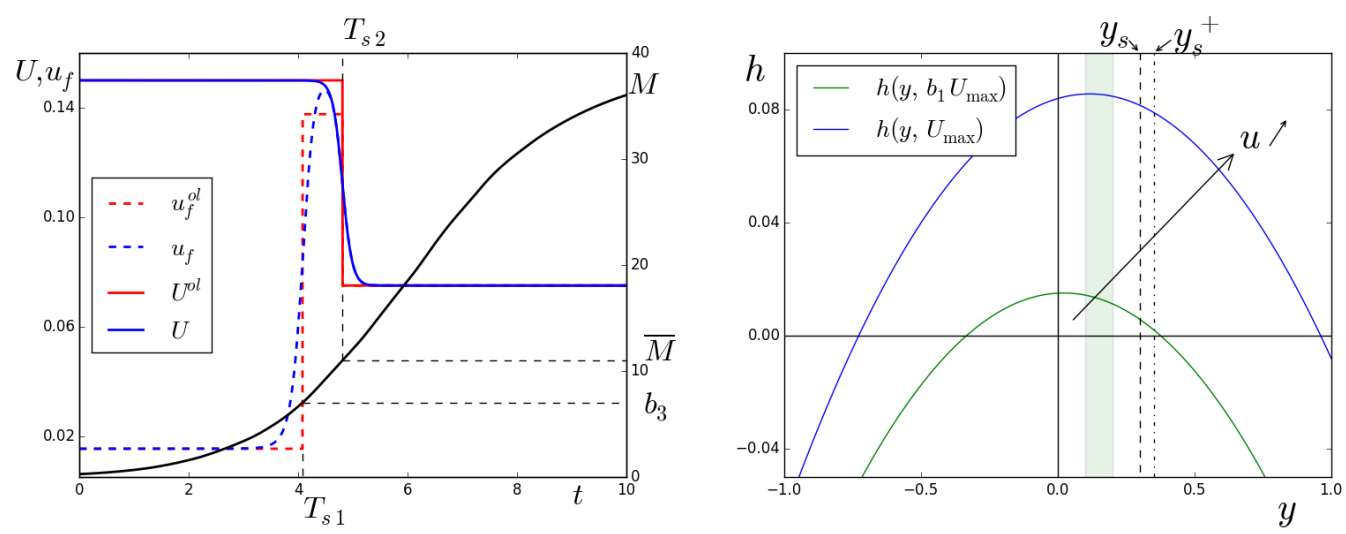

Figure 3.1. Left panel: piecewise constant approximation of the $u_{f}(t)$ input, as defined in Eq. (B.6)-(B.7), characterized by 3 different plateaus (sequentially $b_{1} U_{\max }, \beta_{2} U_{\max }$ and $U_{\min }$ ). Between the 2 switching times $T_{s 1}$ and $T_{s 2}$, the area under the curve is similar in the closed-loop original setup (dashed and solid blue lines) and the approximation (dashed and solid red lines). Right panel: behavior of the maturation function $h(y, u)$, as defined in Eq. (B.2) for extremal control values as a function of maturity $y$ for parameter values $c_{1}=0.3, c_{2}=0.9, \bar{u}=0.1, \tau_{h}=0.12, b_{1}=0.2$, $U_{\max }=0.15$. The green filled area indicates the extremal positions of initial condition $\mu_{1}=0.1$, $\mu_{2}=0.2$.

Since the cell density is spread out over a range of maturity values at initial time, we assume that the maturation velocity remains positive over the whole range of $y$ for the control values that we consider (it is indeed possible to find parameters ensuring these properties, as shown in the right panel of Figure 3.1). Hence the entry (resp. exit) times can be ordered as first entry (resp. exit) time for the cells having the highest initial maturity $\left(\mu_{2}\right)$ and last entry (resp. exit) time for the cells having the lowest initial maturity $\left(\mu_{1}\right)$. Note that, due to the spreading of initial age over the 
whole first cycle, the characteristic times computed from a same initial maturity can be different from one initial age to another.

If $T_{s 1}$ and $T_{s 2}$ are given fixed a priori values, the question raises of how characteristic times computed as explained below from a pair $\left(x_{0}, y_{0}\right)$ are ordered with respect to them. Alternatively, $T_{s 1}$ and $T_{s 2}$ can be defined as being coincident with specific characteristic times and their values assessed accordingly.

To derive the expression of a chosen characteristic time, we will solve one or both of the following elementary problems, enunciated for constant control values, depending on whether the value of $u_{f}$ remains constant all over the trajectory or if we have to take into account a switch in the value of $u_{f}$.

\section{Free time elementary problem $\mathcal{E}_{1}$ :}

Starting from the initial age and maturity $\left(x_{0}, y_{0}\right)$ and given a target maturity $y_{1}$, compute the time $T\left(y_{1}\right)$ needed to reach $y_{1}$, with associated age $x_{1}(T)$

$$
\left(x_{1}, T\right)=\mathcal{E}_{1}\left(y_{1} ; x_{0}, y_{0}, u\right), \quad y_{0}<y_{1} .
$$

\section{Fixed time elementary problem $\mathcal{E}_{2}$ :}

Starting from the initial age and maturity $\left(x_{0}, y_{0}\right)$ and given a fixed time interval $T$, compute the maturity $y_{1}(T)$ and corresponding age $x_{1}(T)$ reached after $T$

$$
\left(x_{1}, y_{1}\right)=\mathcal{E}_{2}\left(T ; x_{0}, y_{0}, u\right) .
$$

3.1. Solving the free time elementary problem. We assume that the aging velocity $g_{f}$ in phase $G 1$ or $D$, is constant between $y_{0}$ and $y_{1}$. We consider two cases Case 1 If $y_{0}<y_{1} \leq y_{s}, g_{f}$ is a positive valued function of the constant control $u_{f}$ (as defined for instance in Eq. (B.6)-(B.7)).

Case 2 If $y_{s} \leq y_{0}<y_{1}, g_{f}=1$.

The case $y_{0}<y_{s}<y_{1}$ can be handled by combining Case 1 to compute the time needed to reach $y_{s}$ (and corresponding $x_{s}$ ), starting from $\left(x_{0}, y_{0}\right)$, with Case 2 to compute the time needed to reach $y_{1}$ (and corresponding $x_{1}$ ), starting from $\left(x_{s}, y_{s}\right)$. In both cases, we can compute the time spent in G1 or D, needed to increase the maturity from $y_{0}$ to $y_{1}$ as

$$
T_{h_{f}}\left(y_{0}, y_{1}\right)=\int_{y_{0}}^{y_{1}} \frac{d y}{h_{f}(y, u)}
$$

In Case $2, T_{h_{f}}$ is directly the solution of the elementary problem, with $x_{1}\left(T_{h_{f}}\right)=$ $x_{0}+T_{h_{f}}$.

In Case 1, things are a little bit more tricky, since the increase in maturity only occurs during phase G1, so that $T_{h_{f}}$ corresponds only to the time spent in successive G1 phases $\left(T_{G 1}\right)$, while the solution time $T$ has also to account for the time spent in phase $S M$, where maturity remains constant (so that the target maturity $y_{1}$ is necessarily reached during phase $G 1)$.

Hence, $T_{G 1}=T_{h_{f}}$ and $T=T_{S M}+T_{G 1}$.

Given that the length of phase $G 1$ is 0.5 and its duration is $t_{G 1}=0.5 / g_{f}$, the number of successive G1 phases covered in $T_{G 1}$ is the integer part $\left\lfloor T_{G 1} / t_{G 1}\right\rfloor$. Each G1 phase is separated from the previous one by a SM phase lasting $t_{S M}=0.5$. The total time $T$ depends on the initial age $x_{0}$, or more precisely the age modulo the length of the cell cycle $\left(D_{c}=1\right), \alpha_{0}=x_{0}-\left\lfloor x_{0}\right\rfloor$ (see left panel of Figure 3.2). 

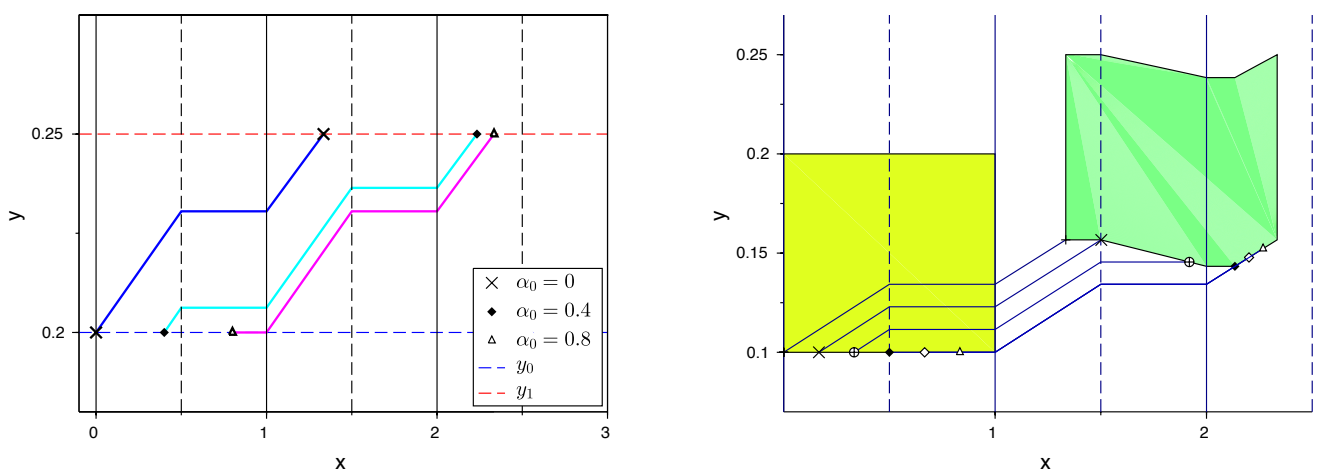

FIGURE 3.2. Left panel: notations and setup for the free time elementary problem with $y_{0}=0.2$ and $y_{1}=0.25$, and $\gamma_{1}=1.48, \gamma_{2}=0.38$ in $g_{f}=\gamma_{1} u+\gamma_{2}$ (such as defined in Eq. (B.1)), and other parameter values defined as in Figure 3.1. The blue path corresponds to a cell starting from phase $G 1$, with $x_{0}=\alpha_{0}=0$, that reaches $y_{1}$ after $T=2.58$ at age $x_{1}=1.33$. The cyan path corresponds to a cell starting from phase $G 1$, with $x_{0}=\alpha_{0}=0.4$, that reaches $y_{1}$ after $T=3.08$ at age $x_{1}=2.23$. The pink path corresponds to a cell starting from phase $S M$, with $x_{0}=\alpha_{0}=0.8$, that reaches $y_{1}$ after $T=2.78$ at age $x_{1}=2.33$. Right panel: density clouds at time 0 (in yellow) and at time $T=2.58$ (in green). Solid lines represent paths followed from different initial ages $x_{0}$ and same maturity $y_{0}=\mu_{1}=0.1$, during the fixed time $T$. The initial and final positions of a given path are materialized with the same symbol. One can see that, if the cells starting from phase $S M$ follow identical trajectories, they stop at different maturities $y$ according to their initial age $x$.

- if $\alpha_{0}<0.5$ the cell starts from phase $G 1$. It takes it $t_{i n i}=\left(0.5-\alpha_{0}\right) / g_{f}$ to complete the remaining part of phase $G 1$, then 0.5 to cover the SM phase of the first cycle. Then the remaining time $\left(T_{G 1}-t_{i n i}\right)$ in phase $G 1$ corresponds to $\left\lfloor 2\left(T_{G 1}-t_{i n i}\right) g_{f}\right\rfloor$ complete G1 phases and as many complete SM phases, plus a remaining uncomplete G1 phase

$$
\begin{aligned}
T\left(x_{0}, y_{0}, y_{1}\right) & =T_{G 1}+0.5\left(1+\left\lfloor 2\left(T_{G 1}-t_{i n i}\right) g_{f}\right\rfloor\right) \\
& =T_{G 1}+0.5\left(1+\left\lfloor 2 T_{G 1} g_{f}-1+2 \alpha_{0}\right\rfloor\right)
\end{aligned}
$$

The corresponding age is

$$
x_{1}=T_{G 1} g_{f}+0.5\left(1+\left\lfloor 2 T_{G 1} g_{f}-1+2 \alpha_{0}\right\rfloor\right)+x_{0}
$$

- if $0.5 \leq \alpha_{0}<1$ the cell starts from phase $S M$. It takes it first $1-\alpha_{0}$ to reach the beginning of the next cycle. Then it covers the $T_{G 1}$ in $G 1$ which corresponds to $\left\lfloor\frac{T_{G 1}}{t_{G 1}}\right\rfloor=\left\lfloor 2 T_{G 1} g_{f}\right\rfloor$ complete $G 1$ phases and as many complete SM phases

$$
T\left(x_{0}, y_{0}, y_{1}\right)=T_{G 1}+1-\alpha_{0}+0.5\left\lfloor 2 T_{G 1} g_{f}\right\rfloor
$$

The corresponding age is

$$
x_{1}=T_{G 1} g_{f}+1-\alpha_{0}+0.5\left\lfloor 2 T_{G 1} g_{f}\right\rfloor+x_{0}
$$

3.2. Solving the fixed time elementary problem. In the case where $y_{1}<y_{s}$ (as illustrated on right panel of Figure 3.2), the time $T$ can be broken down as before into the sum of the times spent in several G1 and SM phases

$$
T=T_{G 1}+T_{S M}=D_{G 1} / g_{f}+D_{S M} .
$$


The distance $D_{G 1}$ (resp. $\left.D_{S M}\right)$ covered all along $G 1$ (resp. $S M$ ) depends on the starting position in the cycle

$$
\begin{aligned}
& \text { if } 0 \leq \alpha_{0}<0.5, D_{G 1}=k / 2-\alpha_{0}+\min \left(\alpha_{1}, 0.5\right), D_{S M}=k / 2+\left(\alpha_{1}-0.5\right)^{+} \\
& \text {if } 0.5 \leq \alpha_{0}<1, D_{G 1}=(k-1) / 2+\min \left(\alpha_{1}, 0.5\right), D_{S M}=(k+1) / 2-\alpha_{0}+\left(\alpha_{1}-0.5\right)^{+}
\end{aligned}
$$

where $\alpha_{1}=x_{1}-\left\lfloor x_{1}\right\rfloor, k=\left(\left\lfloor x_{1}\right\rfloor-\left\lfloor x_{0}\right\rfloor\right) \in \mathbb{N}$, and $x_{1}$ is the unknown.

There is a 4 case combinatorial choice for the $\left(\alpha_{0}, \alpha_{1}\right)$ pair, depending on whether the starting and arrival position lie in phase $G 1$ or $S M$. After some algebra, we end up with

1. if $0 \leq \alpha_{0}<0.5$

$$
\begin{aligned}
& k=\lfloor x\rfloor, \text { with } x=\frac{2\left(T g_{f}+\alpha_{0}\right)}{1+g_{f}} \\
& \alpha_{1}= \begin{cases}\frac{\left(1+g_{f}\right)(x-k)}{2} & \text { if } k \leq x<k+\frac{1}{1+g_{f}} \\
\frac{\left(1+g_{f}\right)(x-k)+g_{f}-1}{2 g_{f}} & \text { if } k+\frac{1}{1+g_{f}} \leq x<k+1 .\end{cases}
\end{aligned}
$$

2. if $0.5 \leq \alpha_{0}<1$

$$
\begin{aligned}
& k=\lfloor x\rfloor, \text { with } x=\frac{2 g_{f}\left(T+\alpha_{0}-1\right)+1-g_{f}}{1+g_{f}} \\
& \alpha_{1}= \begin{cases}\frac{\left(1+g_{f}\right)(x-k)}{2} & \text { if } k \leq x<k+\frac{1}{1+g_{f}} \\
\frac{\left(1+g_{f}\right)(x-k)+g_{f}-1}{2 g_{f}}+1 & \text { if } k+\frac{1}{1+g_{f}} \leq x<k+1 .\end{cases}
\end{aligned}
$$

From $k$ and $\alpha_{1}$, we derive $x_{1}=\left\lfloor x_{0}\right\rfloor+k+\alpha_{1}$, compute $D_{G 1}$ using (3.11) or (3.12), and then $T_{G 1}=D_{G 1} / g_{f}$. We obtain $y_{1}$ by inverting the integral equation (3.5) and substituting $T_{h_{f}}=T_{G 1}$.

If the resulting value $y_{1}$ is such that $y_{1} \leq y_{s}$, we are done (see an illustration on Figure $3.2)$.

If $y_{1}>y_{s}$, we have to proceed in 2 steps. First we compute from the free time elementary problem the time $T_{e}$ taken to reach $y_{s}$ and the corresponding age $x_{s}\left(T_{e}\right)$. Then we solve the fixed time elementary problem with fixed time $T-T_{e}$ and starting point $\left(x_{s}, y_{s}\right)$, by substituting $T-T_{e}$ instead of $T_{G 1}$ in (3.5).

The case $y_{0} \geq y_{s}$ is treated similarly by using (3.5) with $T$ instead of $T_{G 1}$.

3.3. Combining the two elementary problems. We can now compute any characteristic time $T_{P+A}, T_{e}$ and $T_{D+A}$ from given initial conditions, and more specifically:

$T_{P+A}(\mu)$, defined as the minimum time taken by cells starting from a given maturity $\mu$ to reach the lowest boundary $y_{s}^{-}$of the apoptosis zone

$$
T_{P+A}(\mu)=\left\{\begin{aligned}
\min _{x}\left\{T_{1},\left(x_{1}, T_{1}\right)=\mathcal{E}_{1}\left(y_{s}^{-} ; x, \mu, b_{1} U_{\max }\right)\right\} & \text { if } T_{P+A} \leq T_{s_{1}} \\
T_{s_{1}}+\min _{x}\left\{T_{1},\left(x_{1}, T_{1}\right)=\mathcal{E}_{1}\left(y_{s}^{-} ; x_{0}, y_{0}, \beta_{2} U_{\max }\right),\right. & \\
\left.\left(x_{0}, y_{0}\right)=\mathcal{E}_{2}\left(T_{s 1} ; x, \mu, b_{1} U_{\max }\right)\right\} & \text { otherwise }
\end{aligned}\right.
$$


$T_{e}(\mu)$, defined as the maximum time taken by cells starting from a given maturity $\mu$ to reach the differentiation threshold $y_{s}$

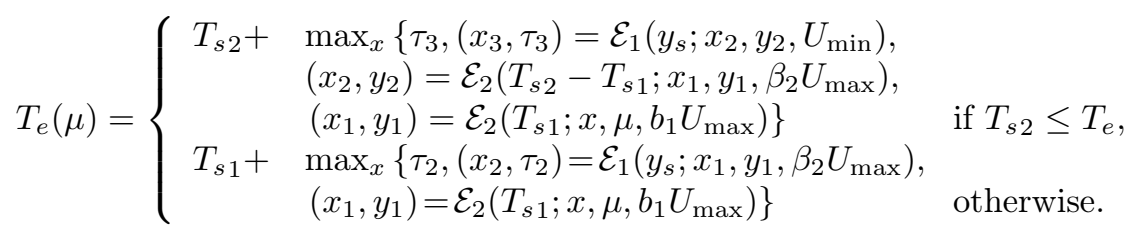

In both cases, the computation has to take into account the possible changes in the control value if $T_{s 1}$ (and possibly $T_{s 2}$ for $T_{e}\left(\mu_{1}\right)$ ) occurs before the target maturity is reached. The characteristic time $T_{D+A}\left(\mu_{1}\right)$, defined as the maximum time taken by cells starting from the lowest maturity $\mu_{1}$ to reach the upper bound of the apoptosis area $y_{s}^{+}$, is computed similarly as $T_{e}\left(\mu_{1}\right)$.

Remark This particle-like approach only deals with time and the cell position within the spatial domain, but it skips out the cell number, since particles are not endowed with any weight linked to the local cell number. Yet, a rough approximation of the time evolution of the global cell number can be derived from assessing the number of complete cell cycles performed by a single particle starting from an average (and median, since the distribution in maturity is uniform) initial maturity $\left(\mu_{1}+\mu_{2}\right) / 2$ :

$$
m_{f}^{0}(t)=M_{0} 2^{\left\lfloor\frac{a(t)}{D_{c}}\right\rfloor}=M_{0} 2^{\lfloor a(t)\rfloor}, \text { for } t \leq T_{e} .
$$

\section{Application to the calibration of the original model.}

4.1. Calibration of a single ovulatory trajectory. To perform numerical simulations of the model, we have to instance the numerical values of the parameters listed in Table C.1 that either delimit the shape and size of the computation domain, enter the formulation of the mathematical functions involved in the dynamics, or define the initial conditions. In this section, we will detail how we can either fix nominal values for some of the parameters or impose constraints on the bounds of parameter ranges.

4.1.1. Domain geometry: $\mathrm{x}_{\mathbf{s}}, \mathbf{D}_{\mathbf{c}}, \mathrm{y}_{\mathbf{s}}, \mathrm{y}_{\mathbf{s}}^{-}, \mathrm{y}_{\mathbf{s}}^{+}, \mathbf{y}_{\max }$. The dynamics of a follicle's trajectory is mostly characterized by the transit times within the different phases of the computation domain. As explained in the previous section, the transit time from the initial to the maturity threshold $y_{s}$ controls the instant of cell cycle exit, hence the maximum cell count; similarly, the transit time within the apoptotic zone controls the cumulative exposure to apoptosis, hence the cumulative cell loss.

For rather obvious computation purposes, the degrees of freedom that we use to control the transit times both horizontally and vertically are the aging and maturation velocities, while the length and height of the domain subparts are kept constant and can be set to any arbitrary value. Hence, the geometry of the computation domain is fixed once for all, with the additional constraints that the domain subparts are paved by an integer number of meshes and that the same discretization step is used in both direction $\Delta x=\Delta y$. Choosing as unit length the duration of the cell cycle, $D_{c}$, we thus have $y_{\max }=1$, and, for the sake of simplicity, we set $x_{s}=y_{s}=1 / 2$ (this amounts to multiplying the time by a scaling factor (here $2 / 3$ ) to recover the physical time scale in days, for an average cell cycle duration of one day).

Also, the boundaries of the apoptotic zone, $y_{s}^{-}$and $y_{s}^{+}$should coincide with horizontal boundaries between two consecutive meshes. From the hypotheses underlying the 
choice of $\Lambda(y, U)$, we can consider that the support of this function is rather narrow, and represents one tenth of the height of phase G1 and D on either side of the $y=y_{s}$ boundary. Setting the values of $y_{s}^{-}$and $y_{s}^{+}$imposes the value of $\bar{y}$.

4.1.2. Specification-based constraints on the parameters. From the bibliographic corpus dedicated to terminal follicular development, we can draw-up a list of specifications that should be fulfilled either by the parameters or some model outputs, as detailed in Appendix C.2. Part of these specifications are summarized in a dataset that will be used as such, in the optimization part of the calibration strategy (see next sections) or as a guide to define the checklist that any ovulatory trajectory should fulfill.

We can also readily define the initial conditions. First, we consider that the distribution in age covers the whole first cell cycle and that it takes into account the discontinuities in the aging velocity on the internal boundaries as defined by equation (2.8). For the distribution in maturity, the upper bound is defined from the expected time taken by a cell with initial maturity $\mu_{2}$ to reach the apoptotic zone (start of FSHdependency), while the lower bound is defined from the expected time taken by a cell with initial maturity $\mu_{1}$ to exit the cell cycle (time of stabilization in the cell number). Finally, the total cell number is fixed from the pseudo-data set to $\approx 210^{5}$ cells.

Finally, we can propose bounds for the parameters in the velocity functions. The derivation of these bounds is detailed in Appendix B.2, we just comment here the underlying principles. First, the velocities are functions of the control term $u_{f}(t)$, whose values cover a closed interval determined by the bounds of $U(t)$ and the parameter of the (closed loop or piecewise constant version of) $b_{f}$ function, so that we can restrict the study to the admissible values of $u_{f}(t)$. Second, there are specific assumptions on the roots of $h_{f}$ needed to ensure that the velocity is positive in the proliferative part of the domain $\left(r_{2}(u, \gamma)>\gamma_{s}\right.$ for all admissible $\left.u\right)$ and to discriminate the ovulatory trajectories from the atretic ones in a FSH-poor environment. Lastly, the modulation operated by the control term on the aging velocity in phase $G 1$ is moderate and is fixed by a modulation coefficient of $r \%$ (set to $10 \%$ in practice).

4.1.3. Characteristic time based calibration of an ovulatory trajectory. The fallouts of subsections 4.1.1 and 4.1.2, combined with Appendix C.2 and B.2 provide us with:

1. Explicit a priori fixed values for the geometric parameters (delimiting subdomains inside the computation domain: $\left.D_{c}=1, y_{s}^{-}=0.45, y_{s}=0.5, y_{s}^{+}=0.55\right)$ as well as the bounds of the global control $\left(U_{\min }=0.075, U_{\max }=0.150\right)$.

2. Implicit a priori constraints relating respectively some parameters of the aging (horizontal) velocity $g_{f}\left(\gamma_{1}, \gamma_{2}\right)$ and maturation (vertical) velocity $h_{f}$ $\left(c_{1}, c_{2}, \bar{u}\right)$ with some of the parameters of the transfer function $b(f)\left(b_{1}, b_{2}\right.$, in the closed-loop case or $b_{1}, \beta_{2}$, in the open-loop case). Implicit means here, that when instantiating a first initial guess for either the ovulatory or atretic follicles (respectively before step 1 and at step 4 of the procedure summarized in Figure C.2 in Appendix C.4) or at each iteration of the (CMAES-based) optimization steps (see steps 1, 2, 4 and 5 in Figure C.3), one first choose (randomly or not) the values of the corresponding 4-uplets or 5 -uplets ${ }^{\dagger}$, and then check that the corresponding $\gamma_{1}, \gamma_{2}$ (resp. $c_{1}, c_{2}$ ) values do belong to the delineate close set $\Omega_{g}$ (resp. $\left.\Omega_{h}\right)$ in plane $\left(c_{1}, c_{2}\right)$ (resp. plane $\left(\gamma_{1}, \gamma_{2}\right)$ ).

\footnotetext{
${ }^{\dagger}\left[\gamma_{1}, \gamma_{2}, b_{1}, b_{2}\right]$ and $\left[c_{1}, c_{2}, \bar{u}, b_{1}, b_{2},\right]$ in the closed-loop case, $\left[\gamma_{1}, \gamma_{2}, b_{1}, \beta_{2}\right]$ and $\left[c_{1}, c_{2}, \bar{u}, b_{1}, b_{2}\right]$ in the open-loop case
} 
3. Explicit a priori constraints (fixed bounds) on the remaining parameters. Even if we have fixed bounds or even given values for several parameters, the dimension of the parameter space remains large with 13 unknown parameter values (see the size of $\tilde{p}$ in Table C.2). As a consequence, the direct optimization-based estimation of these parameters is very costly, as each trial requires a 2D Finite Volume simulation. To circumvent this problem, we design a multi-step method that first considers the open-loop situation with control variables $U^{o l}$ and $u_{f}^{o l}$ defined in (3.1) and (3.2). This allows us to perform the identification of a slightly reduced set of 9 parameters (see the size of $p^{\star}$ in Table C.2) with little a priori information, thanks to the low computing time cost of the particle-like model. When we switch to the closed-loop situation, we benefit from an appropriate initial guess for 8 of the parameters (the values of $\hat{p}$ in Table C.2 except $T_{s 2}$ ), so that the $2 \mathrm{D}$ FV-based identification remains tractable.

Appendix C.3.1 details the expressions of the multi-objective functions used along the different calibration steps and describes the practical algorithm implemented to seek local minima by means of the CMAES Python library [10]. An instance of an ovulatory trajectory is presented in the top panels of Figure 4.1. The macroscopic outputs of the models (cell number and follicular maturity) are similar in the open loop (dashed lines) and closed loop (solid lines) situations.
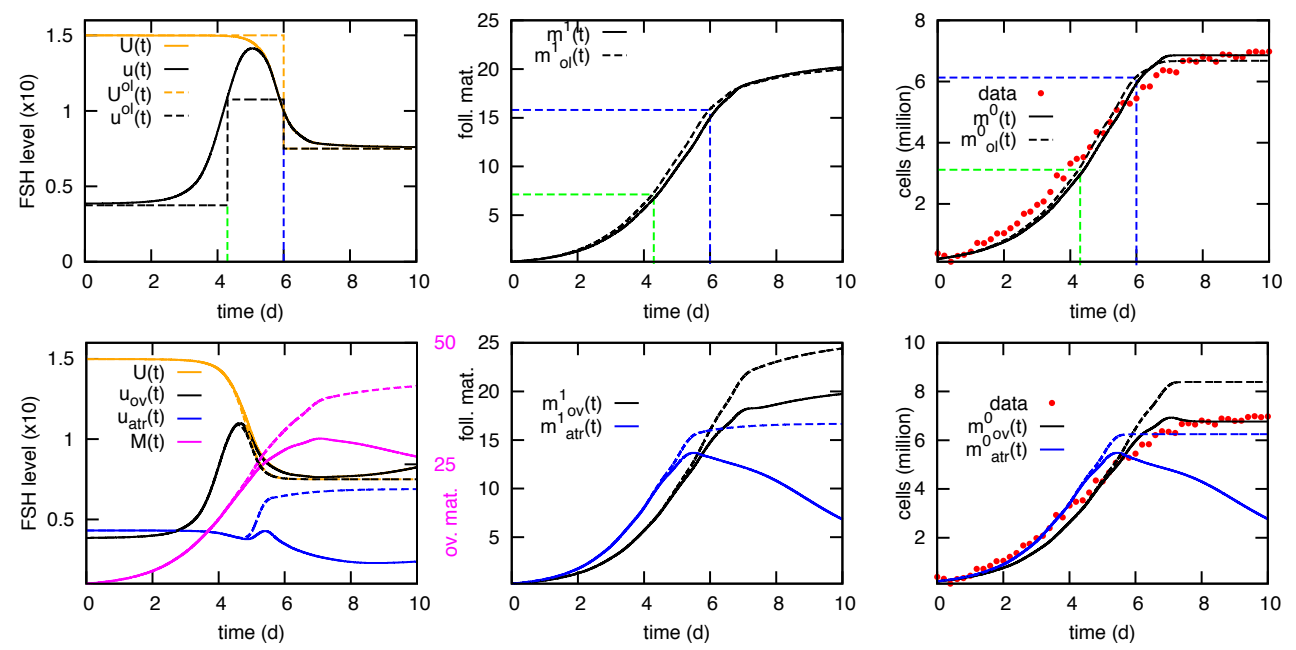

Figure 4.1. Single ovulatory follicle (top panels) and pair of atretic and ovulatory follicles (bottom panels). Left panels: control values, center panels: follicular maturities, right panels: cell numbers. In the single follicle case dashed lines correspond to the open loop situation and solid lines to the closed loop one. In the top panels, the green and blue dashed line correspond respectively to $T_{s 1}$ and $T_{s 2}$. In the bottom panels, the blue and black dashed lines correspond to alternative trajectories when the apoptosis rate is deactivated $(\lambda=0$ in Eq. (B.5)).

4.2. Calibration of interacting ovulatory and atretic trajectories. We finally dispose of a reasonable parameter set $p_{o v}$ (see values in Table C.2) for a closedloop ovulatory trajectory. We now consider the interaction between several follicles, resulting in a situation with ovulatory follicles and atretic ones. Starting with a pair of interacting follicles we first couple the ovulatory trajectory with another trajectory corresponding to an atretic follicle: another multi-objective function, formulated in Appendix C.3.2, is introduced to ensure that for the atretic follicle, the cell number increases significantly with respect to its initial value, up to the selection time, while 
the cell loss becomes substantial after this time, with a final cell number clearly lower than the maximal number reached along the atretic trajectory. In the same time, the penalization of the atretic follicles should not be performed at the expense of a non-realistic level of cell loss in the ovulatory follicles.

It is why, although they are not as directly observable as other macroscopic quantities such as the number of viable cells, we have introduced the instantaneous number of cells undergoing apoptosis-induced death at a given time $d_{f}(t)$ as well as the cumulative number of apoptotic cells all along the follicular trajectory $D_{f}(t)$ :

$$
d_{f}(t)=\iint_{\Omega} \Lambda(x, y, U(t)) \phi_{f}(t, x, y) \mathrm{d} x \mathrm{~d} y, \quad D_{f}(t)=\int_{0}^{t} d_{f}(s) \mathrm{d} s,
$$

given that, in the ovulatory follicle, $D_{f}(t)$ is constrained to remain below a threshold. Then, we design a realistic cohort of $N_{f}$ follicles: for a given number of ovulations $N_{o v}$, we couple trajectories obtained by $N_{o v}$ (respectively $N_{f}-N_{o v}$ ) random fluctuations about the ovulatory (resp. atretic) parameter set and adapt the global parameters in functions $U(t)$ and $\Lambda(t)$.

An instance of an ovulatory/atretic pair is presented in the bottom panels of Figure 4.1 (solid lines). To visualize the contribution of the apoptotic process in the dynamics, the same pair is also followed in the case when the apoptosis rate is deactivated $(\bar{\lambda}=0$, dashed lines); the selection is then not operated and the former atretic follicle becomes able to ovulate.

Figure 4.2 displays the macroscopic outputs for a cohort of 10 follicles. The random perturbations are uniformly distributed within $\pm 10 \%$ of the parameters in Table C.1. The ovulation time $T_{o v}$ is defined as the time when the cumulated ovarian maturity $\int_{0}^{T_{o v}} M(s) d s$ (magenta dashed line in Figure 4.2) has reached a level sufficient to trigger ovulation (840 in this simulation). The follicles are sorted amongst ovulatory follicles (black, blue and green solid lines) and atretic ones (dashed lines). After $T_{o v}$ all follicles whose maturity reaches a theshold (here 15) within one day ovulate.

The top panels display a simulation where all 10 follicles start with the same normalized cell number. At ovulation time $T_{o v}=8.0$ only follicles 1 to 3 have reached a sufficient maturity to ovulate. Follicle 4 (dashed blue line) is yet just below the threshold. The bottom panels display the situation where follicles 4 and 5 start with an enhanced cell number of 1.25 instead of 1 for the seven other follicles. The ovulation time occurs a bit earlier $\left(T_{o v}=7.8\right)$ and by that time both follicles 4 and 5 have managed to reach the ovulatory stage.

5. Discussion. In this work, we have tuned the quantitative behavior of a multiscale model coupling cell kinetics with population dynamics, and spanning scales from the intracellular level to the tissue (ovarian follicle) level. The passage from microscopic to macroscopic dynamics is based, for a single follicle, on the numerical computation of moments and derived outputs such as cell kinetics indexes (growth fraction and mitotic index) and the total cell number. In addition, the connections between scales are not limited to an averaging approach, but they are rather two-way, since the macroscopic interactions between follicles, mediated by the pituitary-ovarian loop, feedback onto the microscopic level.

To calibrate the model parameters, we have investigated thoroughly the links between cell kinetics indexes and the fine distribution of cells within the different cell states (proliferating or differentiated) and cell cycle phases; in some sense, we revisit and extend a corpus of biomathematical works that were undergone at the golden age 

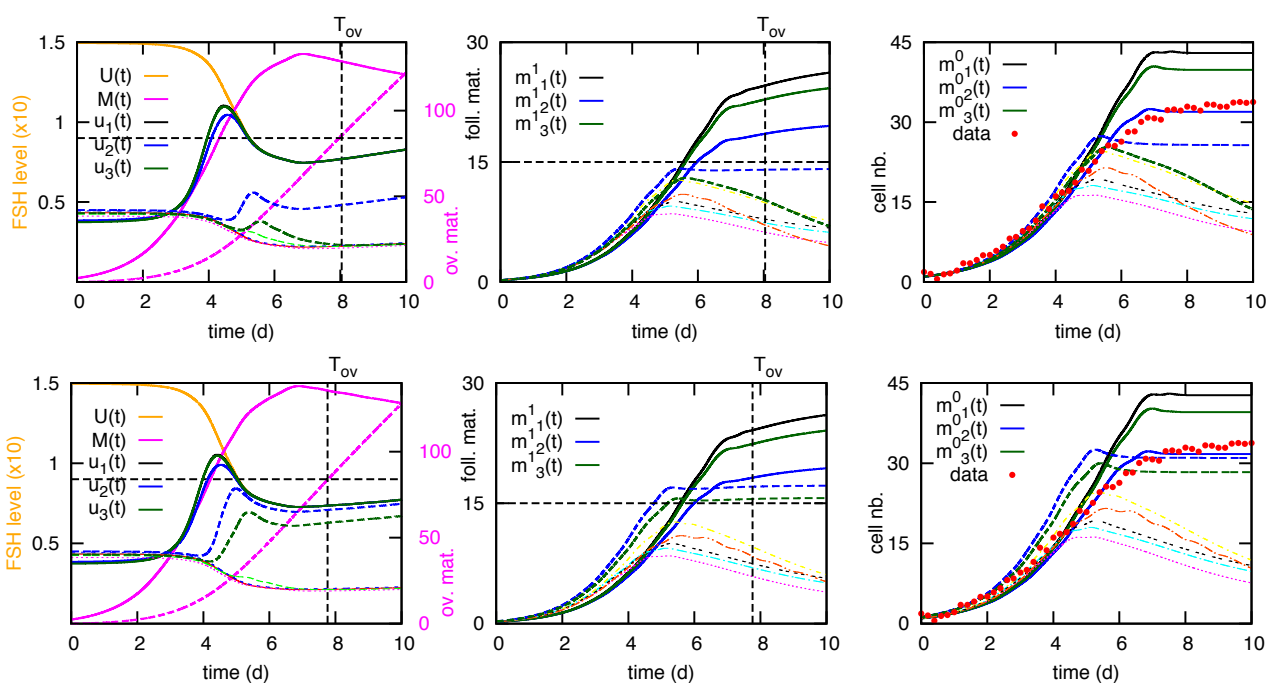

Figure 4.2. Cohort of 10 interacting follicles. Left panels: control values, center panels: follicular maturities, right panels: cell numbers. Trajectories of follicles 1, 2, and 3 (respectively 4 to 7) result from random perturbations applied to the ovulatory (resp. atretic) follicle in Figure 4.1. Compared to top panels, follicles 4 and 5 in bottom panels (dashed green and blue lines) start with a cell number increased by $25 \%$. Dashed black lines indicate the ovulation thresholds. The magenta dashed line is the time integral of $M(t)$ (scaled by 10 for the sake of lisibility). A movie showing the corresponding changes in the cell density is available as supplementary material.

of experimental and theoretical cell kinetics (in the 1970s, see for instance [1]), that studied the impact of different cell distributions (exponential in case of non-limited growth, uniform in case of strict population renewal, possibly subject to cell loss) on the interpretation of experimental cell-kinetics approaches. We have benefited from both the richness of our 2D-structuring of the cell population (versus only an agestructuring) and the power of numerical simulations to tackle more complex situations including time-dependent and even unknown-dependent progression along the cell cycle (see Section A.3 in Appendix A).

At this stage, the biomathematical approach is somehow a step forward on compared to the experimental possibilities, since it is still very difficult to obtain quantitative information on cell dynamics in physiological conditions. For instance, even "simple" cell counting represents a hard-working process, and most of the time, there are not true kinetic data, since, due to the invasive character of the measurements, different experimental subjects correspond to the different time points (as often in physiological issues, time is thus a statistical reconstruction in this case). Yet, one may hope that cutting edge techniques allowing one to visualize the cell cycle distribution in living cells [18], that requires the transfection of cells, will also be easily deployable in more physiological contexts, or that time-lapse monitoring of cell fate (see e.g. [12, 15]) will be usable on larger time and space scales to embed single cell fates within a tissue dynamics.

From the reproductive biology viewpoint, our results have shown that the hormonal control is at the source of selection, since there is no predestination of follicles, neither on the proliferative capital issue (all follicles can potentially reach a similar total cell number at the end of development) nor on the apoptosis issue (any follicle will escape from the apoptosis zone when exposed to a constantly favorable environment). 
With minimal follicular heterogeneity in the parameters affecting only the velocities (and possibly as a first trial instance initial conditions), and a quite narrow range of hormonal (FSH) levels, selection can nevertheless be operated through the model. We have already mentioned and investigated in former studies the importance of managing the proliferative resource of the follicles and controlling the transit time in the apoptosis zone, on the level of a single follicle [7]. One critical point that has been further unraveled by the current study, on the level of the population of interacting follicles, is that there is a relatively little room for manoeuvre to meet at the same time the constraints that (i) the ovulatory follicle(s) should not have a too high cumulated loss of cells and (ii) the atretic follicle should in contrast be subject to a significant cell loss.

We have performed the numerical illustration and parameter fitting of the model in the ewe due to the variety of data sources available in the ovine species, and also because this species exhibits a natural (and genetically-encoded) variability in the ovulation number, that is challenging both on the experimental side and the modeling one. The principle of our approach could be generalized to other species and especially to the human (mono-ovulating) species. We intend to extend the present model of terminal follicular development to women. From the mathematical viewpoint, this extension would not raise new bottlenecks. Yet, from the biomathematical, databased viewpoint, it is not straightforward, since it requires to design a specification list, as we did for the ewe, from very composite information that need to be both gathered and tallied from literature articles dealing with morphologic (monitoring of follicle growth through ultrasonography), histologic, cell kinetics and endocrine issues.

To perform the parameter tuning, we have had to cope with the structural lack of quantitative and kinetic data on in-vivo follicular development. Everything would be much simpler if datasets giving the cell numbers of both ovulatory and atretic follicles against time were available. We thus had to develop a more indirect strategy that brute-force fitting and we could not proceed only through optimization steps. The core of our strategy is the computation of characteristic reaching times in the spatial domain from a particle-like reduction of the model presented in section 3. Compared to previous particle or characteristic-curve based approaches, we focus here on the effect of the drift dynamics in phase SM (pure horizontal transport without any vertical motion) in amplifying the desynchronization between cells sharing the same initial maturity (vertical ordinate) but not the same initial location within the cell cycle. This question amounts to investigating the effect of the cell insensitivity to extracellular signals after the G1-S checkpoint. The formulation of the model from particle systems had already been used in [8] and [6] but in a more simple framework in which either the phase-dependent contrast in the aging velocity, or the heterogeneity in initial conditions had been discarded. A thorough analysis of the model based on the characteristic curves had also been conducted in [19] without any simplifying assumptions, but in a rather theoretical objective (existence and uniqueness of weaksolutions) that is not straightforward to handle numerically especially in the context of inverse problems.

The drawback of the calibration strategy is that at this stage, we cannot assess our parameter sets from the viewpoint of identifiability; we rather provide a set of compatible parameters. Issues of identifiability and statistical estimation could be studied through intensive simulations with random selection of the parameter values; the idea would be to recover for each parameter a distribution compatible with all the specifications, which would provide us with information on the heterogeneity and variability 
in the parameters with respect to different physiological (mono- or poly-ovulation) or pathological (dysovulation or anovulation) situations.

\section{References}

REFERENCES

[1] W.A. Aherne, R.S. Camplejohn, and N.A. Wright. An introduction to cell population kinetics. Edward Arnold, 1977.

[2] B. Aymard, F. Clément, F. Coquel, and M. Postel. Numerical simulation of the selection process of the ovarian follicles. ESAIM Proc., 28:99-117, 2012.

[3] B. Aymard, F. Clément, F. Coquel, and M. Postel. A numerical method for transport equations with discontinuous flux functions: Application to mathematical modeling of cell dynamics. SIAM J. Sci. Comput., 35:6, 2013.

[4] B. Aymard, F. Clément, and M. Postel. Adaptive mesh refinement strategy for a non conservative transport problem. ESAIM Math. Model. Numer. Anal., 90:4, 2014.

[5] D. Bresch, T. Colin, E. Grenier, B. Ribba, and O. Saut. Computational modeling of solid tumor growth: The avascular stage. SIAM J. Sci. Comput., 32(4):2321-2344, 2010.

[6] F. Clément, J.-M. Coron, and P. Shang. Optimal control of cell mass and maturity in a model of follicular ovulation. SIAM J. Control Optim., 51(2):824-847, 2013.

[7] F. Clément and D. Monniaux. Multiscale modelling of follicular selection. Prog. Biophys. Mol. Biol., 113(3):398-408, 2013.

[8] N. Echenim, F. Clément, and M. Sorine. Multi-scale modeling of follicular ovulation as a reachability problem. Multiscale Model. Simul., 6(3):895-912, 2007.

[9] N. Echenim, D. Monniaux, M. Sorine, and F. Clément. Multi-scale modeling of the follicle selection process in the ovary. Math. Biosci., 198:57-79, 2005.

[10] N. Hansen and A. Ostermeier. Adapting arbitrary normal mutation distributions in evolution strategies: The covariance matrix adaptation. In Evolutionary Computation, 1996., Proceedings of IEEE International Conference on, pages 312-317. IEEE, 1996.

[11] N.R. Hartmann and Pedersen T. Analysis of the kinetics of granulosa cell populations in the mouse ovary. Cell Tissue Kinet., 3(1):1-11, 1970.

[12] W. Haubensak, A. Attardo, W. Denk, and W.B. Huttner. Neurons arise in the basal neuroepithelium of the early mammalian telencephalon: A major site of neurogenesis. Proc. Natl. Acad. Sci. U.S.A., 101(9):3196-3201, 2004.

[13] T. Maekawa and J. Tsuchiya. A method for the direct estimation of the length of G1, S and G2 phase. Exp. Cell Res., 53:55-64, 1968.

[14] P. Michel. Multiscale modeling of follicular ovulation as a mass and maturity dynamical system. Multiscale Model. Simul., 9:282-313, 2011.

[15] S.C. Noctor, V. Martínez-Cerdeõ, L. Ivic, and A.R. Kriegstein. Cortical neurons arise in symmetric and asymmetric division zones and migrate through specific phases. Nature Neurosci., $7(2): 136-144,2004$.

[16] B. Perthame. Transport Equations in Biology. Birkhaüser Verlag, Basel, 2007.

[17] M. Rotenberg. Transport theory for growing cell populations. Journal of Theoretical Biology, 103(2):181 - 199, 1983.

[18] A. Sakaue-Sawano, H. Kurokawa, T. Morimura, A. Hanyu, H. Hama, H. Osawa, S. Kashiwagi, K. Fukami, T. Miyata, H. Miyoshi, T. Imamura, M. Ogawa, H. Masai, and A. Miyawaki. Visualizing spatiotemporal dynamics of multicellular cell-cycle progression. Cell, 132(3):487 $-498,2008$.

[19] P. Shang. Cauchy problem for multiscale conservation laws: Application to structured cell populations. J. Math. Anal. Appl., 401(2):896-920, 2013.

[20] C.L. Smith and P.P. Dendy. Relation between mitotic index, duration of mitosis, generation time and fraction of dividing cells in a cell population. Nature, 193:555, 1962. 


\section{Appendix A. Computation of the age distribution within the cell cycle and related cell kinetics indexes in simplified frameworks.}

A.1. Simple exponential cell dynamics. We illustrate here different model outputs in the simplest case of a fully desynchronized and oproliferating population. Figure A.1 shows some snapshots of both the $\phi(t, x, y)$ density (color-code based panels) and the $\psi(t, x)$ density (graphs with red curves), in the simplified 2D framework. In the case of a single cell cycle with periodic boundary conditions (top panels in Figure A.1), the age distribution is the exponentially decreasing in age and increasing in time law (2.3). In the case of two (or more) successive cycles (bottom panels in Figure A.1), the shape of the density is modified at the time of the transition between the consecutive cycles, since the cells are in between the two cycles, and then goes back to the simple exponential pattern once all cells have exited the first cell cycle and lie in the second one. Figure A.2 displays the corresponding macroscopic outputs in the total cell number $m_{f}^{0}(t)$ (eq. 1.8) and mitotic index $M I(t)$. As expected, the cell number is doubled after one cell cycle, while the numerical MI (2.2) remains constant and equal to the theoretical value expected from (2.4).
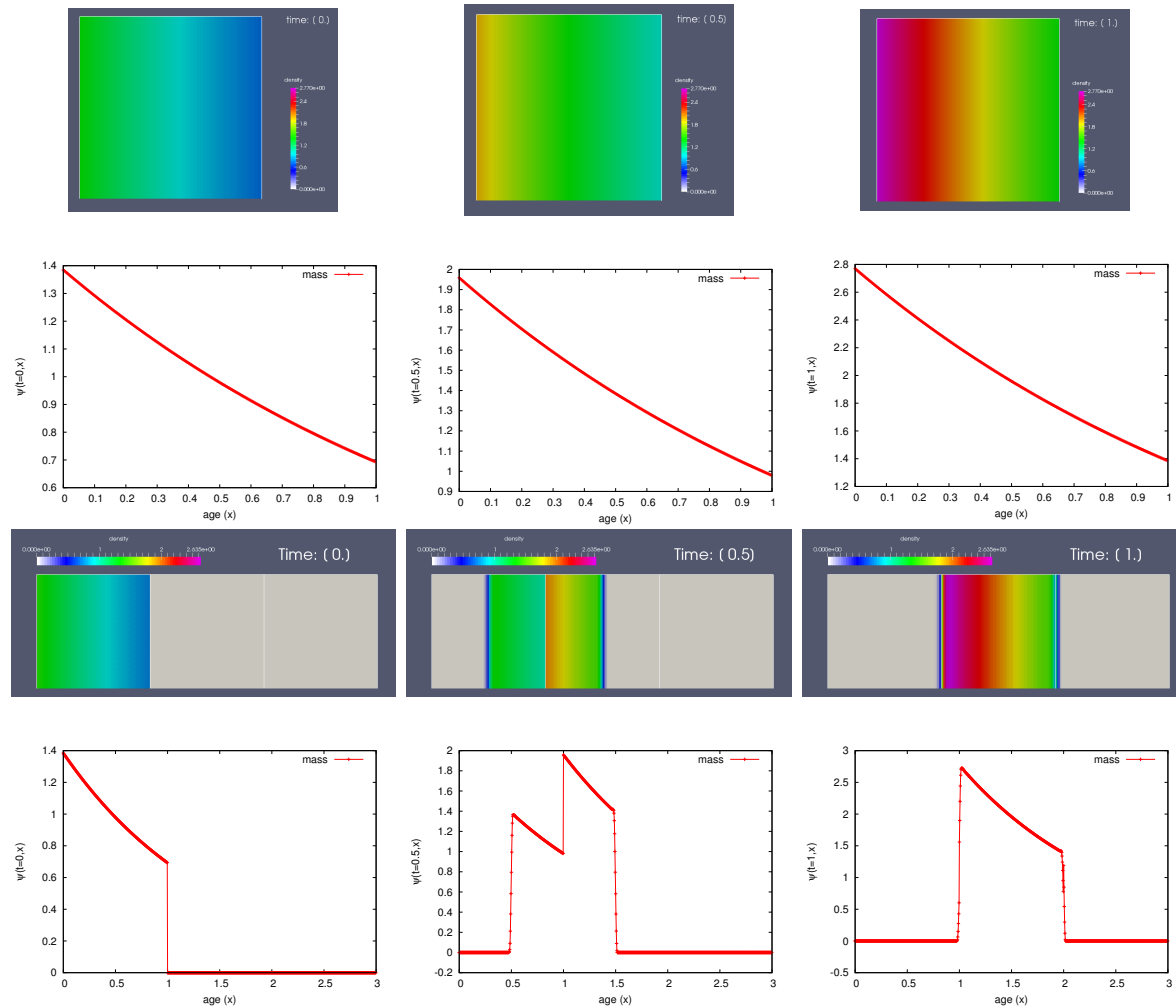

Figure A.1. Snapshots of the cell density $\phi(t, x, y)$ over the $2 D$ domain (color-code panels) and age density $\psi(t, x)$ (red curves) in the case of a fully proliferating, desynchronized cell population, with constant velocity $(g=1)$ of progression along the cell cycle. Top panels: a single cell cycle with periodic boundary conditions. Bottom panels: 2 subsequent cell cycles. Left panels: $t=0.0$, middle panels: $t=0.5$, right panels $t=1$.

A.2. Recursive computation of the MI. We recall briefly here how to compute the solution to equation (2.2) for a series of $N_{c}$ cycles (hence $2 N_{c}-1$ interfaces), 

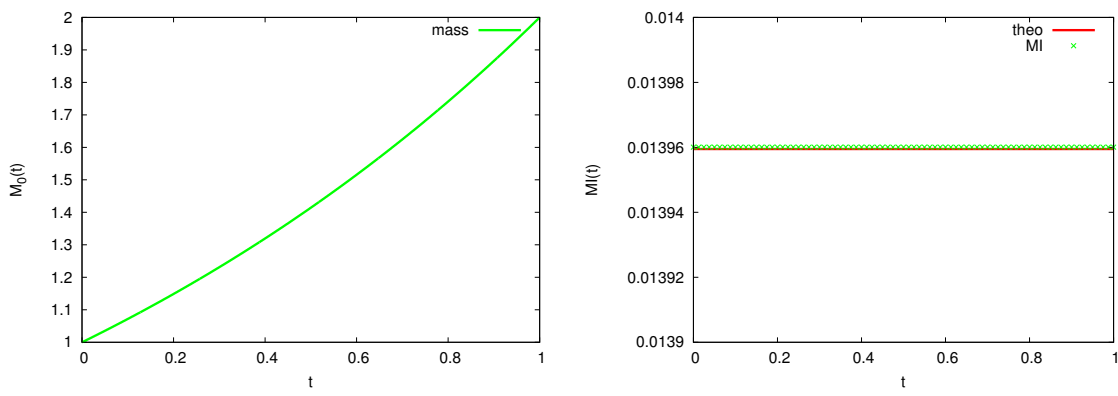

Figure A.2. Macroscopic outputs corresponding to the microscopic outputs of Figure A.1: total cell number $m_{f}^{0}(t)$ (left panel) and mitotic index $M I(t)$ (right panel).

with $D_{c}=2 x_{s}$. On the $p$ th interface located at $x=p \times x_{s}$ the condition on the flux reads:

$$
v_{p} \psi\left(t, p x_{s}^{+}\right)=k_{p} v_{(p-1)} \psi\left(t,(p-1) x_{s}^{-}\right)
$$

where $v_{p}$ is the (constant) velocity on the right of the interface and $v_{(p-1)}$ the velocity on its left, $k_{p}=1$ in the case of flux continuity (interface G1-SM), while $k_{p}=2$ in the case of flux doubling (interface SM-G1 phase where mitosis occurs). For the first interface (and starting the numbering of cell phases from 0 ),

$$
\psi_{1}(t, x)= \begin{cases}\psi_{0}\left(x-v_{0} t\right) & \text { for } \quad x<x_{s} \\ \tilde{\psi}_{1}\left(t_{s}\right) \text { for } \quad\left(x_{s}-v_{1} t\right) \leq\left(x-v_{1} t\right)<x_{s}, \\ \psi_{0}\left(x-v_{1} t\right) \quad \text { for } \quad\left(x-v_{1} t\right) \geq x_{s}\end{cases}
$$

where $\tilde{\psi}_{1}$ is the trace of the solution on the right of the interface, defined by

$$
\tilde{\psi}_{1}(t)=\frac{1}{v_{1}} k_{1}\left(v_{0} \psi_{0}\left(x_{s}-v_{0} t\right)\right)
$$

and $t_{s}$ is the delay after which the effect of interface $x_{s}$ is felt at position $x$ and time $t$ defined by

$$
t_{s}=t-\frac{\left(x-x_{s}\right)}{v_{1}}
$$

We now show how the exact solution for a sequence of $N_{c}$ cell cycles can be defined recursively. Let us denote by $\psi_{p-1}(t, x)$ the solution for a sequence of $p-1$ interfaces, with $p>1$ and add a $p^{\text {th }}$ interface at $p x_{s}$ with a coefficient $k_{p}$ for the transmission condition and a velocity $v_{p}$ on the right hand side. For $x<p x_{s}$ the effect of the $p^{t h}$ interface is not felt and the solution is $\psi_{p-1}(t, x)$. For $x \geq p x_{s}$, depending on time $t$, the effect of the interface is felt or not. If $\left(x-p x_{s}\right)<v_{p} t$, traces of the solution crossing the $p^{t h}$ interface are multiplied by the $k_{p}$ coefficient and transported at velocity $v_{p}$. The effects of these events are felt at position $x$ after a delay $\left(x-p x_{s}\right) / v_{p}$. If $\left(x-p x_{s}\right)>$ $v_{p} t$, the effect of the $p^{t h}$ interface is not felt yet and the initial condition defined on $x>p x_{s}$ is transported at velocity $v_{p}$. We eventually have

$$
\psi_{p}(x, t)=\left\{\begin{array}{l}
\psi_{p-1}(x, t) \quad \text { for } \quad x<p x_{s} \\
\tilde{\psi}_{p}\left(t_{s}^{p}\right) \quad \text { for } \quad\left(p x_{s}-v_{p} t\right) \leq\left(x-v_{p} t\right)<p x_{s} \\
\psi_{0}\left(x-v_{p} t\right) \quad \text { for } \quad x-v_{p} t \geq p x_{s}
\end{array}\right.
$$



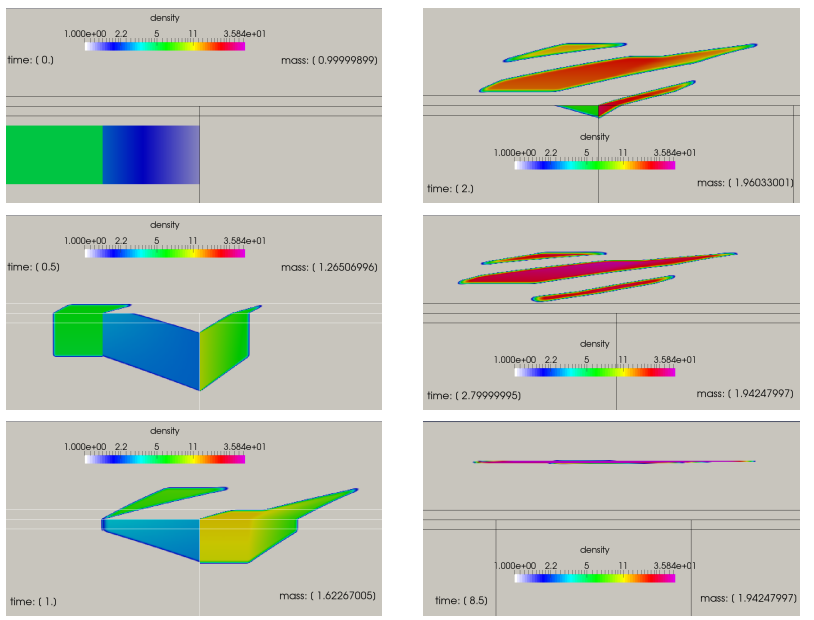

Figure A.3. Snapshots of the cell density $\phi(t, x, y)$ over the $2 D$ domain at $t=0,0.5,1,2,2.7$ and 8.5. Parameters are set to $\tau_{h}=0.4, c_{1}=0.01, c_{2}=1.2, \bar{u}=0.1, g=0.5$ in G1 for the velocities, $U_{\max }=0.15, U_{\min }=0.075$ and $T_{s}=2$ for the open-loop control and $\lambda=0.2, \bar{y}=0.1$ for the apoptosis rate. The same logarithmic color scale is used for all times, from 1 in white to 35.8 in pink. The three horizontal black lines on each snapshot materialize the thresholds $y_{s}^{-}=0.45, y_{s}=0.5$ and $y_{s}^{+}=0.55$. The same logarithmic color code is used for all snapshots. It is well suited to the exponential increase in the cell number and concentration of the density in the maturity direction. Sharp changes in color happen on the interfaces between phases, where velocity contrasts induce density discontinuities due to conditions (1.3), (1.4) and (1.5). The narrowing of the density support in phase $D$ rather induces smooth changes in color, towards the highest color levels. An animated version of this figure is provided as a supplemental movie file.

where $\tilde{\psi}_{p}$ is the trace of the solution on the right of the $p^{t h}$ interface, defined by

$$
\tilde{\psi}_{p}(t)=\left\{\begin{array}{l}
\frac{1}{v_{p}} k_{p} v_{p-1} \psi_{p-1}\left(p x_{s}, t\right) \text { if } p>1 \\
\frac{1}{v_{p}} k_{p} v_{p-1} \psi_{0}\left(x_{s}-v_{p-1} t\right) \text { otherwise }
\end{array}\right.
$$

and $t_{s}^{p}$ is defined by

$$
t_{s}^{p}=t-\frac{\left(x-p x_{s}\right)}{v_{p}} .
$$

A.3. Instance of a detailed simulation in the case of cell cycle exit induced by differentiation and/or apoptosis. We now comment a detailed simulation corresponding to a dynamical framework that has been designed to embed, as simply as possible, differentation- or apopotosis-induced cell cycle exit, and to study its impact on the model outputs on different scales. The microscopic outputs for the $\phi(t, x, y)$ density are illustrated on Figure A.3, where snapshots are shown at six different times. The computational domain is made of eight consecutive cycles, but for each snapshot, we only display the part of the computational domain containing the support of the density.

At initial time, the density is all contained within the first cell cycle and the maturity range is $[0.1,0.4]$. At time $t=0.5$, some cells have undergone mitosis and spread over the second cycle, while others are exiting the first cell cycle. At time $t=1$, the density is splitting into two disconnected regions; some of the cells have already 
escaped from the apoptosis zone (their maturity overcomes the $y_{s}^{+}$threshold in phase D). At time $t=2.0$, the density has almost completely entered the upper phase of the domain and it is composed of three disconnected regions. The same is true at time $t=2.8$, when all cells have not only exited the cell cycle, but also escaped from the apoptosis zone. The last snapshot, at time $t=8.5$ illustrates the "contracting" property of the maturation function, that tends to pitch the density horizontally as the average maturity approaches the asymptotic value corresponding to the positive root (here $r_{2}\left(U_{m i n}\right)$ ); this property has been theoretically established in [12] for a slightly different model where the distinction between $S M$ and $G 1$ phases is not made.
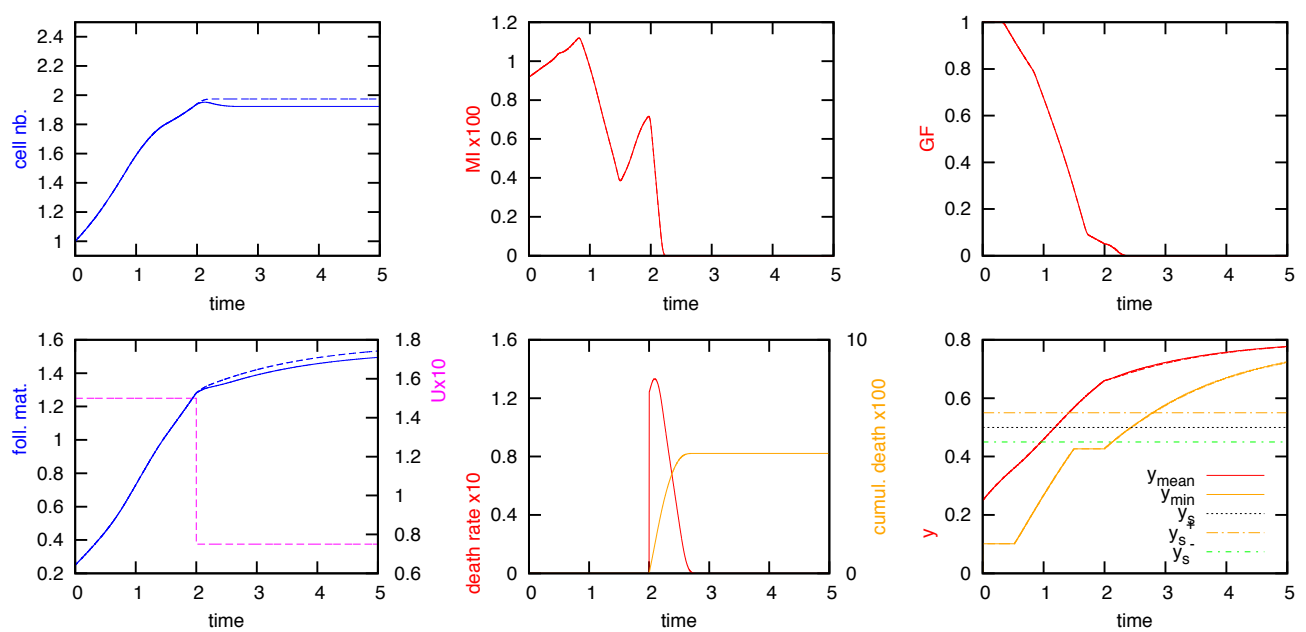

Figure A.4. Macroscopic outputs: total cell number (top left panel) ; mitotic index (top center panel) ; growth fraction (top right panel) ; piecewise constant control and follicular maturity (bottom left panel) ; instantaneous and cumulated cell death (bottom center panel); average (red solid line) and minimal (red dashed line) cell maturity (bottom right panel). In the left panels the solid line correspond to $\lambda=1.6$ in the sink term, the dashed line corresponds to $\lambda=0$ (no apoptosis). The effect of apoptosis is hardly visible on the mesoscopic outputs.

The macroscopic outputs are displayed on Figure A.4. In addition to the total cell number (top left panel) and mitotic index (top center panel), the figure shows the decrease in the growth fraction (top right panel) due to cell cycle exit $(G F$ was not shown in the former subsections since $G F=1$ there) and the changes in the follicular maturity $m_{1}^{1}(t)$ (bottom left panel, which was neither shown up to now, because its time pattern superimposed on the total cell number). Also, to understand these macroscopic outputs in more depth, we introduce new mesoscopic indexes, such as (i) the changes in the minimum, average and maximum maturity (bottom right panel), and (ii) the instantaneous and cumulated cell death (bottom center panel). We also plot the changes in $U(t)$ according to (2.9), where $T_{s}=2.0$ to condense all interesting events (and especially the triggering of apoptosis) in short time (the physiological drop would occur later, see next sections).

With the set of parameters chosen for this example, the total cell number stops increasing around $t=2.11$. Before that time, the slope in the cell number increase had been lowered due to he intensification of cell differentiation. The growth fraction decreases steadily from its maximal value $(1$, since all cells are in the proliferative part of the domain at initial time) to a null value at the time when all cells have exited 
the cell cycle. This exit time (around $t=2.33$ ) roughly coincides with the abscissa of the crossing point between the horizontal line delimiting the $y_{s}$ threshold and the minimum maturity (red dashed line on the bottom right panel), even if numerical diffusion blurs a little the precise assessment of the coordinates of this crossing point. It is not exactly the same as that when the total cell number stabilizes, due to effect of apoptosis. A slowing-down in $G F$ decrease can be seen between $t=1.7$ and $t=2.11$. This is due to a transient relative lowering of the denominator in (1.9) with respect to its numerator. After $t=2.0$, apoptosis is active and is no more compensated by proliferation. Indeed the cell number even decreases a little before stabilizing around $t=2.54$. The mitotic index first follows a pattern resembling that of Figure 2.1 with a sharply damped second peak, and then drops to zero at the exit time. 


\section{Appendix B. Complementary information on model-specific formulation and parameterization.}

B.1. Specific formulation of the aging and maturation velocities and their control. Both the aging and velocity functions $g_{f}(x, y, u)$ and $h_{f}(x, y, u)$ are space-dependent ; their expressions depend either directly on the space variables, or indirectly, through the location within a given subpart of the domain. They also take as argument a control variable, that can be defined either as an open-loop function of time (see $u_{f}^{o l}$ in 3.2) or as a closed-loop function defined from some moments of the cell density.

The aging function $g_{f}$ appearing in (1.1) is defined by

$$
g_{f}(x, y, u)= \begin{cases}\gamma_{1}^{f} u+\gamma_{2}^{f} & \text { for }(x, y) \in \Theta_{1} \\ 1 & \text { for }(x, y) \in \Theta_{2} \cup \Omega_{3}\end{cases}
$$

where $\gamma_{1}^{f}, \gamma_{2}^{f}$ are real positive constants. The aging velocity $g_{f}$ modulates the transit time along phase $G 1$ and its relative duration with respect to the total cell cycle duration $D_{c}$. Hence, even if phase $G 1$ is assumed to have a different duration than the remaining of the cycle (phase $S M$ ), we can set $x_{s}$ to $D_{c} / 2$ without loss of generality ; the lengths of $G 1$ and $S M$ will be the same on the domain, but their relative duration will be dynamically settled by the aging function and the choice of its parameter values. In practice, we deal with a phase $G 1$ longer than $S M$, so that the aging velocity will be lower than 1 .

The maturation function $h_{f}$ is defined by

$$
h_{f}(x, y, u)= \begin{cases}\tau_{h}^{f}\left(-y^{2}+\left(c_{1}^{f} y+c_{2}^{f}\right)\left(1-\exp \left(\frac{-u}{\bar{u}^{f}}\right)\right)\right) & \text { for }(x, y) \in \Theta_{1} \cup \Omega_{3} \\ 0 & \text { for }(x, y) \in \Theta_{2}\end{cases}
$$

where $\tau_{h}^{f}, c_{1}^{f}, c_{2}^{f}$ and $\bar{u}^{f}$ are real positive constants. An important feature of $h_{f}(x, y, u)$ is that for fixed $u$, it possesses only one strictly positive root in $y$, and this root remains below a maximal threshold as the value of $u$ increases. Let us denote

$$
\theta(u)=1-\exp -\frac{u}{\bar{u}^{f}}, \quad \theta(0)=0, \text { and } \lim _{u \rightarrow \infty} \theta(u)=1 .
$$

The roots $r_{i}(u)_{i=1,2}$ of $h_{f}$ read:

$$
r_{i}(u)=\frac{1}{2}\left(c_{1}^{f} \theta(u) \pm \sqrt{\left(c_{1}^{f} \theta(u)\right)^{2}+4 c_{2}^{f} \theta(u)}\right)
$$

It is easy to check that the positive root $r_{2}(u)$ increases with $u$ :

$$
r_{2}(0)=0 \text { and } \lim _{u \rightarrow \infty} r_{2}(u)=\frac{1}{2}\left(c_{1}^{f}+\sqrt{c_{1}^{f^{2}}+4 c_{2}^{f}}\right) .
$$

The sink term, that represents cell loss through apoptosis, is defined by

$$
\Lambda(x, y, U)= \begin{cases}\lambda \exp \left(-\left(\frac{y-y_{s}}{\bar{y}}\right)^{2}\right) \times \frac{U_{\max }-U}{U_{\max }} \mathbb{1}_{\left[y_{s}^{-}, y_{s}^{+}\right]} & \text {for }(x, y) \in \Theta_{1} \cup \Omega_{3} \\ 0 & \text { for }(x, y) \in \Theta_{2}\end{cases}
$$

where $\lambda, y_{s}$ and $\bar{y}$ are real positive constants. $\Lambda(x, y, U)$ is nonzero only in a restricted range $y \in\left[y_{s}^{-}, y_{s}^{+}\right]$with $0<y_{s}^{-}<y_{s}<y_{s}^{+}<y_{\max }$, while its maximal value is reached 
when both $U$ takes its lowest value $U_{\min }$ (penalization due to poor hormonal supply), and $y=y_{s}$ (highest sensitivity to apoptosis at the cell cycle exit transition).

The $F$ equations in the PDE system (1.1) are linked together through the arguments $u_{f}(t)$, appearing in the velocities $g_{f}(x, y, u)$ and $h_{f}(x, y, u)$, and $U(t)$, appearing in the sink term $\Lambda(x, y, U)$, which depend on the first maturity moment of the densities. The plasma FSH level $U(t)$ showing up in the arguments of the sink term in (1.1) is defined from the global maturity (see 1.7) by

$$
\begin{aligned}
U(t) & =S(M(t)) \\
\text { with } S(M) & =U_{\min }+\frac{U_{\max }-U_{\min }}{\left(1+\exp (c(M-\bar{m}))^{\delta}\right)}
\end{aligned}
$$

where $U_{\min }, U_{\max }, c, \delta$ and $\bar{m}$ are real positive constants.

The locally bioavailable FSH level $u_{f}(t)$ showing up in the arguments of the velocities in (1.1) is defined by

$$
\begin{aligned}
u_{f}(t) & =b_{f}\left(m_{f}^{1}(t)\right) U(t) \quad \text { for } f=1, \ldots, F, \\
\text { with } b_{f}(m) & =b_{1}^{f}+\frac{1-b_{1}^{f}}{1+\exp \left(-b_{2}^{f}\left(m-b_{3}^{f}\right)\right)},
\end{aligned}
$$

where $b_{1}^{f}, b_{2}^{f}$ and $b_{3}^{f}$ are real positive constants. The sigmoid shapes of $S(M)$ and $b_{f}(m)$ ensure altogether that the mesoscopic control $u_{f}$ is bounded

$$
b_{1}^{f} U_{\min } \leq u_{f}(t) \leq U_{\max } .
$$

To alleviate the notations, we drop from now on the upper index $f$ in the constants $\gamma_{1}^{f}$, $\gamma_{2}^{f}, \tau_{h}^{f}, c_{1}^{f}, c_{2}^{f}, \bar{u}^{f}, b_{1}^{f}, b_{2}^{f}$ and $b_{3}^{f}$, which indicates that they can vary from one follicle to another. The follicle-dependent character of these parameters will be reminded in the sequel by the $f$ index in functions $g_{f}, h_{f}$ and $u_{f}$. We now comment on the outer boundaries of the computing domain. From (B.4) and (B.8), we can see that the maturation velocity in the $y$ direction vanishes at some ordinate lying below a supremum $r_{2}\left(U_{\max }\right)$ and above an infimum $r_{2}\left(b_{1} U_{\min }\right)$. Choosing $\mu_{2}, c_{1}, c_{2}$ such that

$$
\mu_{2} \leq y_{s}<r_{2}\left(b_{1} U_{\min }\right) \text { and } \frac{1}{2}\left(c_{1}+\sqrt{c_{1}^{2}+4 c_{2}}\right)<y_{\max }
$$

ensures that $h_{f}(y,$.$) is strictly positive until above y_{s}$ and negative before reaching $y_{\max }$, so that the density remains null on these two boundaries at all times. This enables us to use periodic boundary conditions in the numerical implementation (see [2]).

\section{B.2. Derivation of constraints for the parameters of the aging and mat- uration velocities.}

Bounds of the parameters entering the $g_{f}$ aging function. Here we detail how we fix the bounds of the set $\Omega_{g}$ of admissible values for the $\gamma_{1}$ and $\gamma_{2}$ parameters in the aging velocity function $g_{f}$

$$
\Omega_{g} \subsetneq\left[0, \gamma_{1 \max }\right] \times\left[\gamma_{2 \min }, g_{\max }\right]
$$

Let us denote by $\left[u_{\min }, u_{\max }\right]$ the extremal values that may be taken by the local control $u_{f}$. In the open loop piecewise constant setup (3.2)

$$
u_{\min }=b_{1} U_{\max }, \quad u_{\max }=\max \left(b_{2} U_{\max }, U_{\min }\right),
$$


while in the closed loop original setup (B.6,B.7)

$$
u_{\min }=b_{1} U_{\min }, \quad u_{\max }=U_{\max } .
$$

From the aging velocity and the length of the cell phases, we can derive the average duration of phases SM and G1

$$
T_{S M}=\frac{D_{c}-x_{s}}{1}, \quad \text { and } T_{G 1}=\frac{x_{s}}{g_{f}},
$$

Denoting by $r \%$ the level of modulation operated by the hormonal control on the aging velocity, we get

$$
\frac{T_{S M}}{T_{G 1}}(1-r) \leq g_{\min }<g_{f}\left(u_{f}(t)\right)<g_{\max } \leq 0.5(1+r) \frac{T_{S M}}{T_{G 1}}
$$

In our specific case $D_{c}=2 x_{s}=1$, so that $T_{S M} / T_{G 1}=1 / 2$, and we obtain the following inequality constraints for $\gamma_{1}$ and $\gamma_{2}$

$$
\begin{array}{r}
g_{\min } \leq \gamma_{1} u_{\min }+\gamma_{2} \\
\gamma_{1} u_{\max }+\gamma_{2} \leq g_{\max }
\end{array}
$$

so that

$$
\gamma_{1 \max }=\frac{g_{\max }-g_{\min }}{u_{\max }-u_{\min }} \text { and } \gamma_{2 \min }=\frac{g_{\min } u_{\max }-g_{\max } u_{\min }}{u_{\max }-u_{\min }} .
$$

Note that the bounds in (B.14) depend indirectly on the parameters of the $b_{f}$ function, and more specifically on $b_{1}$ (and also $\beta_{2}$ in the open loop setup). In practice, we first select the pair $\left(\gamma_{1}, \gamma_{2}\right)$ independently, and then check whether the selected values are compatible with all other constraints imposed on the whole parameter set. A practical domain for searching the pair is the minimal box containing $\cup_{b_{1}} \Omega_{g}$, i.e. the bounds (B.14) evaluated at $b_{1}=b_{1 \max }$.
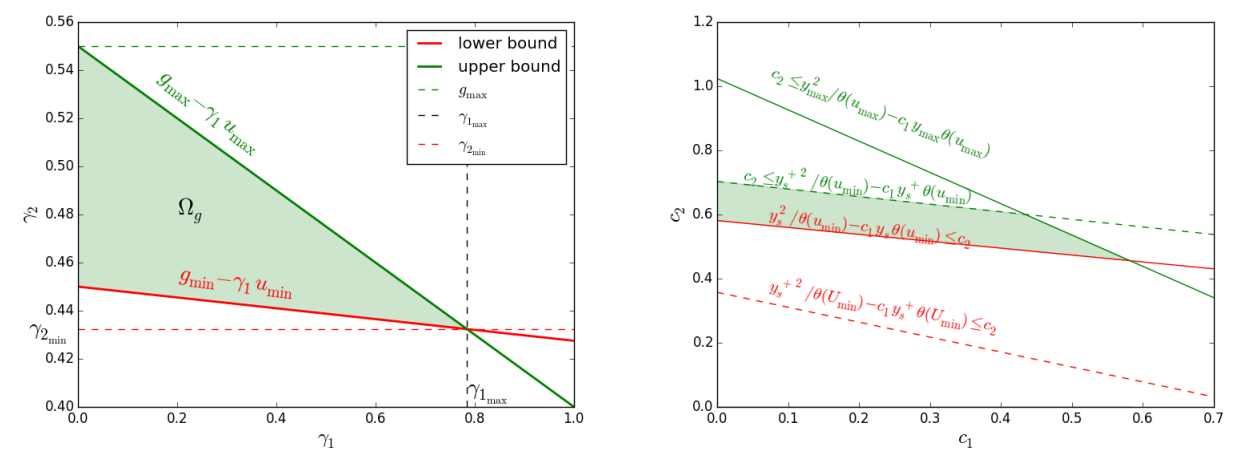

Figure B.1. Left panel : admissible values for parameters $\gamma_{1}, \gamma_{2}$, satisfying constraints (B.13) $\left(g_{\min }=0.45, g_{\max }=0.55, b_{1}=0.3\right.$, closed loop setup $)$. Right panel: admissible values for parameters $c_{1}, c_{2}$, satisfying constraints (B.19). The polygons $\Omega_{g}$ and $\Omega_{h}$ are delimited by the green-filled areas. 
Bounds of the parameters entering the $h_{f}$ maturation function. We will now derive constraints on the bounds of the set $\Omega_{h}$ of admissible values for the $c_{1}$ and $c_{2}$ parameters in the maturation velocity function $h_{f}$

$$
\Omega_{h} \subsetneq\left[0, c_{1 \max }\right] \times\left[c_{2 \min }, c_{2_{\max }}\right]
$$

The positive root of $h_{f}, r_{2}\left(u_{f}\right)$ is an increasing function of $u_{f}$. In the ovulatory case, the development of the follicle is well synchronized with the FSH environment ("right time - right place"), so that it is able to take advantage of even low level of FSH $\left(U_{\min }\right)$ and

$$
\begin{array}{r}
y_{s} \leq r_{2}\left(u_{\min }\right) \\
r_{2}\left(u_{\max }\right)<y_{\max } .
\end{array}
$$

along with

$$
y_{s}^{+} \leq r_{2}\left(U_{\min }\right) .
$$

Yet, if the follicle fails to adapt to low FSH levels [9], $\left(b_{f} \leq 1\right.$ when $\left.U=U_{\min }\right)$, then, as in the atretic case, the cells can be trapped in the part of the differentiated domain where they are sensitive to apoptosis in case of an unfavorable FSH environment (typically when $U(t)=b_{1} U_{\min }$ ) and

$$
y_{s} \leq r_{2}\left(b_{1} U_{\min }\right) \leq y_{s}^{+}
$$

Similarly to $\Omega_{g}$, the bounds of $\Omega_{h}$ depend indirectly on the parameters of the $b_{f}$ function, $b_{1}$ and $\bar{u}$ (as well as $b_{2}$ in the open loop setup). As can be seen on the right panel of Figure B.1, $\Omega_{h}$ is a polygon bounded by the following lines, derived from (B.16,B.17,B.18)

$$
\begin{gathered}
y_{s}^{2} / \theta\left(u_{\min }\right)-c_{1} y_{s} \theta\left(u_{\min }\right) \leq c_{2} \\
c_{2} \leq y_{\max }^{2} / \theta\left(u_{\max }\right)-c_{1} y_{\max } \theta\left(u_{\max }\right) \\
y_{s}^{+2} / \theta\left(U_{\min }\right)-c_{1} y_{s}^{+} \theta\left(U_{\min }\right) \leq c_{2} \leq y_{s}^{+{ }^{2}} / \theta\left(u_{\min }\right)-c_{1} y_{s}^{+} \theta\left(u_{\min }\right)
\end{gathered}
$$

The combinations of $c_{1}$ and $c_{2}$ that fall within the polygon area are not arbitrary and we can derive a condition preventing from emptiness of $\Omega_{h}$

$$
\max \left(\frac{y_{s}^{+^{2}}}{\theta\left(U_{\min }\right)}, \frac{y_{s}{ }^{2}}{\theta\left(u_{\min }\right)}\right) \leq \min \left(\frac{y_{s}^{+^{2}}}{\theta\left(u_{\min }\right)}, \frac{y_{\max }^{2}}{\theta\left(u_{\max }\right)}\right)
$$

When the condition is met

$$
\Omega_{h} \subsetneq\left[0, c_{1 \max }\right] \times \mathbb{R},
$$

with

$$
c_{1 \max }=\max \left(0, \min \left(\frac{\frac{y_{\max }^{2}}{\theta\left(u_{\max }\right)}-\frac{y_{s}^{2}}{\theta\left(u_{\min }\right)}}{y_{\max } \theta\left(u_{\max }\right)-y_{s} \theta\left(u_{\min }\right)}, \frac{y_{s}^{+}+y_{s}}{\theta\left(u_{\min }\right)^{2}}\right)\right)
$$

The existence of non-empty sets is illustrated as a function of parameters $\bar{u}$ and $c_{1}$ in the left panel of Figure B.2. 

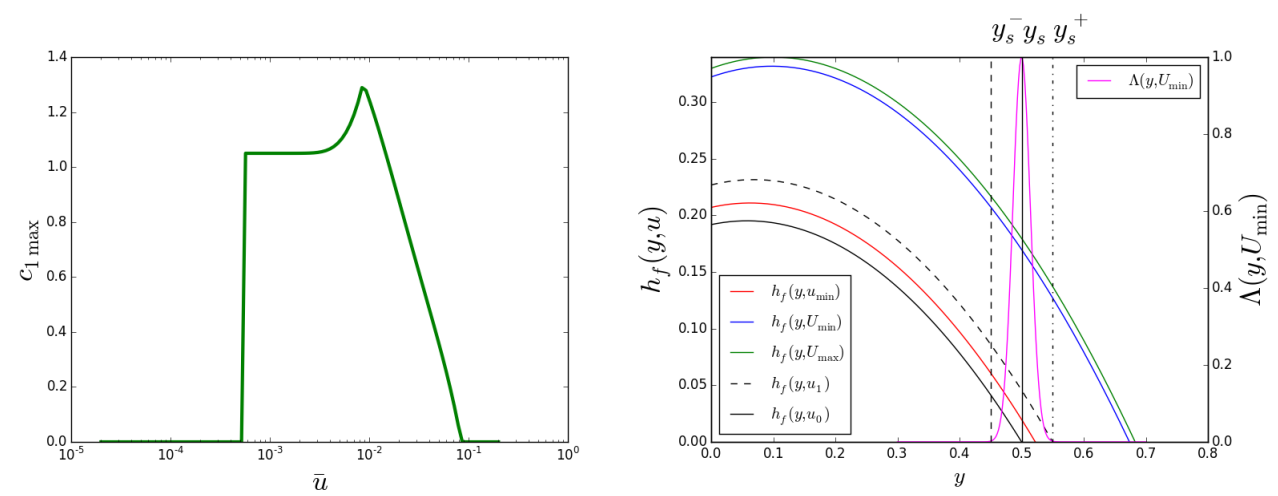

Figure B.2. Left panel: sketch of non emptiness criterium (B.20). Maximum bound for $c_{1}$ parameter as a function of $\bar{u}$. Right panel: $h_{f}(y, u)$ and $\Lambda\left(y, U_{\max }\right)$ versus $y$ for $c_{1}=0.2, c_{2}=0.5$, $\bar{y}=0.02, u=U_{\max }$ (green line), $u=U_{\min }$ (blue line), $u=u_{\min }$ (red), $u=u_{0}$, with $r_{2}\left(u_{0}\right)=y_{s}$ (black solid line) and $u=u_{1}$, with $r_{2}\left(u_{1}\right)=y_{s}^{+}$(black dashed line).
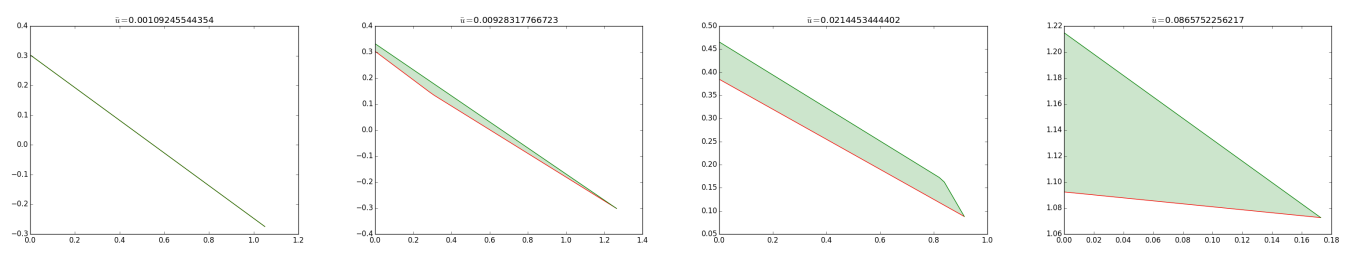

Figure B.3. Admissible sets $\left(c_{1}, c_{2}\right)$ for varying values of $\bar{u}$ (from left to right $0.001,0.009,0.021,0.086)$.

Once we are guaranteed that $c_{1} \in\left[0, c_{1 \text { max }}\right]$, we deduce that

$$
\begin{aligned}
c_{2 \min } & =\max \left(\frac{u_{s}^{+2}}{\theta\left(U_{\min }\right)}-c_{1} y_{s}^{+} \theta\left(U_{\min }\right), \frac{y_{s}^{2}}{\theta\left(u_{\min }\right)}-c_{1} y_{s} \theta\left(u_{\min }\right)\right), \\
c_{2 \max } & =\min \left(\frac{u_{s}^{+2}}{\theta\left(u_{\min }\right)}-c_{1} y_{s}^{+} \theta\left(u_{\min }\right), \frac{y_{\max }^{2}}{\theta\left(u_{\max }\right)}-c_{1} y_{\max } \theta\left(u_{\max }\right)\right) .
\end{aligned}
$$

The shape and area of different admissible sets corresponding to different values of $\bar{u}$ are depicted in Figure B.3, while Figure B.2 gives an instance of the resulting graph of $h_{f}(y, u)$ versus $y$ after applying the strategy for finding a specific parameter set belonging to $\Omega_{h}$. 


\section{Appendix C. Biological foundations and dedicated calibration strategy for a multiscale model of cell population dynamics in ovarian follicles.}

In this appendix, we provide the reader with information that are rather specific to the issue of follicular development modeling.

C.1. Biological and biomathematical background. The ovulation event is the endpoint of the developmental process undergone by ovarian follicles, which are spheroidal tissular structures composed of somatic cells and sheltering the oocyte (the female gamete). The follicular development is a morpho-dynamical process spanning several months and involving both proliferation and differentiation of the follicular cells, as well as enlargement of the follicle due first to the increase in the number of cell layers and then to the creation and dilatation of a fluid cavity (an antrum) inside the follicle [11]. During each ovarian cycle, ovulation results in the release of one (in monoovulating species) or several (in poly-ovulating species or strains) oocyte(s) competent for subsequent fertilization and embryo development. The number of ovulations is determined during the terminal phase of follicular development, as the issue of a selection process regulated by endocrine loops between the ovaries and hypothalamopituitary axis. Hence, the selection of ovulatory follicle(s), and its corollary, the degeneration of the non-selected follicles, caused by the apoptosis of follicular cells, is an original instance of population dynamics coupled with hormonally-controlled cell kinetics.

The multiscale model initially introduced in [7] and presented in section 1 of the main text has inherited some of its characteristic features from the highly complex control of terminal follicular development. First, to account for the differential (yet not uncoupled) control exerted by FSH onto the commitment of its target follicular cells towards either proliferation or differentiation, we have introduced two structuring variables, the age $x$ and maturity $y$, hence we have to deal with a $2 \mathrm{D}$ equation for each of the (possibly numerous) follicles. Second, to account for the feedback exerted by the follicles onto the pituitary (mediated by the contributions of follicular cells to the secretion of estradiol and inhibin by the ovaries), we have introduced control terms (denoted by $U(t)$ and $u_{f}(t)$ in the sequel) underlying the interactions between follicles, whose formulation depends on moments of the unknowns. This amounts to considering FSH-dependent expression of the velocities, and especially of the maturation function, whose biochemical foundations were settled in [6]). An instance of moment-based control term occurs in the context of hematopoiesis (see e.g. [1]). However, in that case, as in other problems of control of hyperbolic partial differential equations (see e.g. [17]), the control term operates on a boundary, whereas in our case the control variables operate on the velocities and sink term. Third, to account for the differences in cell sensitivity to extracellular signals according to the different phases of the cell division cycle [23], we cannot distribute the term representing mitosis (cell doubling) over all cell ages, and we have to tackle discontinuities both in the velocities and density on internal boundaries of the domain representing the passage from one cell phase to another.

C.2. Biological specifications for parameter calibration. All our numerical simulations will be illustrated in the ewe. Indeed, large domestic species, and especially the ovine species, are particularly interesting to investigate the bases of follicular development, since (i) they have a large body size allowing repeated blood sampling and further analysis of endocrine time series, (ii) the duration of the ovarian cycle makes it easier to dissect the different steps in the temporal sequence of hormonal 
feedbacks between the ovaries and hypothalamo-pituitary axis and its links with the follicle selection process, (iii) they are closer to human ovarian physiology compared to rodents, and (iv) there exist in several ewe strains natural mutations affecting the ovulation number, that correspond to different genetic strategies of poly-ovulation [16]. Many experimental studies have been undertaken on follicular development in this species, so that we can access a variety of experimental data ranging from cell kinetics of granulosa cells or in vivo monitoring of follicle growth to endocrine time series of pituitary and ovarian hormones.

From the bibliographic corpus dedicated to terminal follicular development in the ewe, we can draw-up a list of specifications that should be fulfilled either by the parameters or some model outputs. Part of these specifications are summarized in a dataset first introduced in [5], that relates the cell number to the follicular age (red bullets on Figure C.1), by combining data on follicular growth (changes in diameter assessed either by histology [21, 22] or ultrasonography [15]) with data on granulosa cell numbers according to the diameter [20]. The initial age corresponds to $1 \mathrm{~mm}$ in diameter follicles, when ovarian follicles undergo at this size their maximal proliferative activity ([21, 22]) and can be recruited into the selection process [19]. This set of data will be used as such, in the optimization part of the calibration strategy (see next sections) or as a guide to define the checklist that any ovulatory trajectory should fulfill. From this dataset, we can derive constraints on the range of cell values that should be reached at some critical points of follicular development, that are given in the literature with respect to the diameter, and that we can convert in time:

1. the switch from a FSH-responsive to a FSH-dependent status in follicles $2.5 \mathrm{~mm}$ in diameter [16, 13]; from this diameter, the follicles become unable to pursue their development further if they are not supplied with FSH (as it has been shown in many occasions of surgical or pharmacological hypophysectomy);

2. the selection of the future ovulatory follicles around $4 \mathrm{~mm}$ in diameter ; at this diameter, the drop in the growth fraction is the highest [14], as is the sensitivity towards FSH, as assessed by the rate of follicle degeneration [16]; dominant follicles are "saved" thanks to their new ability to transfer their gonadotropin dependency from FSH to LH (luteinizing hormone);

3 . the ovulation at an ovulatory size around $7-7.5 \mathrm{~mm}$ in the mono-ovulatory strains of sheep ; at this size, one can assess the ratio of increase $C C$ in the cell number from the initial cell number at diameter $1 \mathrm{~mm}$ :

$C C \in\left[C C_{\min }, C C_{\max }\right] \approx[25,35]$; since in the ovulatory case the cell number starts stabilizing as soon as the maturity of all cells overcomes $y_{s}^{+}$, this ratio also gives a rough indication on the bounds of the global maturity of the ovarian follicle from selection to ovulation: $m_{f}^{1}(t) \in\left[C C_{\min } y_{s}^{+}, C C_{\max } y_{\max }\right]$.

Besides these specifications, the bounds of the $S(M(t))$ function given in equation (B.6) can be set in a more straightforward way, since they can be read from time series of FSH plasma levels along the ovarian cycle (see e.g. [15, 18]). During the time horizon spanned by the model, the FSH level starts from its maximum and drops by half at the end of the follicular phase. Hence we can set $U_{\min }=U_{\max } / 2$. We can also get some a priori idea on the location of the inflexion point ruled by the value of $\bar{m}$ and consider that

$$
C C_{\min } y_{s}^{+} \leq \bar{m}_{\min } \leq \bar{m} \leq \bar{m}_{\max } \leq C C_{\max } y_{\max }
$$

which amounts to assume that the dominant follicle contributes the most to the FSH 


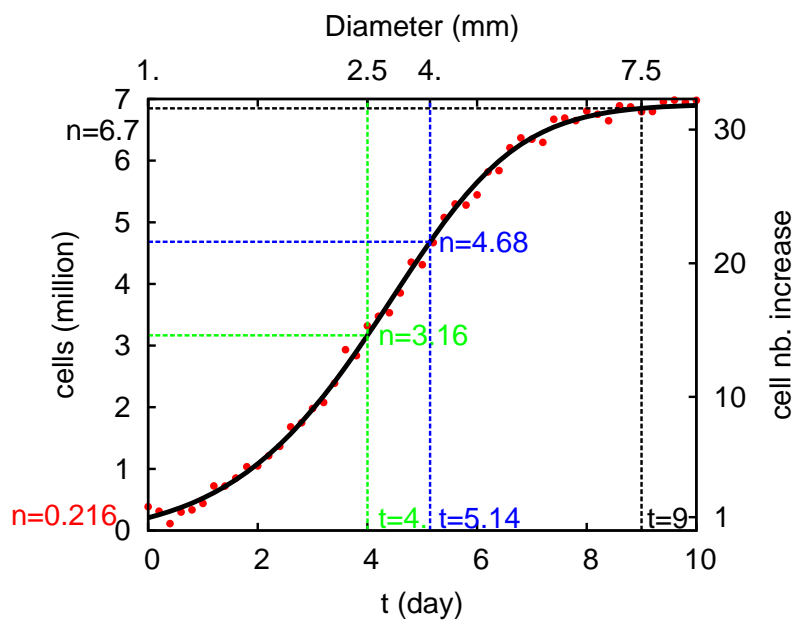

Figure C.1. Specifications on the cell number in an ovulatory follicule. The cell number is shown both as a function of diameter (top horizontal axis) and time (bottom horizontal axis). From the initial cell number $(\approx 0.216), 3$ checkpoints can be defined from the biological knowledge and applied to our training data set (red bullets superimposed on the solid black line); we expect to reach given cell numbers at the time when the follicle switches from a FSH-responsive to a FSH-dependent status (green marks, diameter $2.5 \mathrm{~mm})$, when it switches from a mainly proliferative $(G F>0.5)$ to a mainly differentiated status (blue marks, diameter $4 \mathrm{~mm}$ ) and when it reaches the ovulatory size (diameter $7.5 \mathrm{~mm}$ ). The cell number is illustrated in physical unit (million of cells) on the left vertical axis, and as the ratio of increase from the normalized initial number on the right vertical axis.

drop.

It is not straightforward at all to define similar specifications in the case of atretic follicles, since there are numerous ways for a follicle to follow an an atretic trajectory. Yet, we recall here some additional comments on the hypotheses underlying the choice of formulation of $\Lambda(y, U)$, that are related to specifications for atretic follicles (the shape of $\Lambda$ is displayed in the right panel of Figure B.2). Indeed, the formulation of the cell loss intensity transcribes on the cell level what is well established on the follicle level. Indeed, the vulnerability of a follicle to atresia (follicle degeneration) is maximal at the time of selection of future ovulatory follicles (follicles of $3-4 \mathrm{~mm}$ in ewes [13]), which is also the time when the drop in the growth fraction is the highest. Before the selection time, this vulnerability appears at the moment of the switch from a FSH-responsive to a FSH-dependent stage (follicles of $2,5 \mathrm{~mm}$ in ewes [16]), while it disappears when the follicle is selected (dominant follicle) and able to switch its dependence from FSH to LH. Since follicle atresia is mediated by granulosa cell apoptosis, it seems quite natural to draw an equivalence between the cell sensitivity to apoptosis and the follicle dependence towards FSH, and between, respectively, (i) the exit time (where $y=y_{s}$ ) and the selection time, (ii) the time when the cell reaches $y_{s}^{-}$ and the time of FSH-dependency triggering and (iii) the time when the cell overcomes $y_{s}^{+}$and the time of dominance. 
C.3. Optimization steps using CMAES and flow charts for the whole procedure of parameter calibration. In this section, we explain in details the procedure reviewed in section 4.1.3 and whose final output is a complete set of parameter values for the original model, given all a priori constraints on the values or bounds of the parameters (as summarized in Table C.1).

\begin{tabular}{|l|c|c|}
\hline \multicolumn{3}{|c|}{ Global parameters } \\
\hline Model & Name & Value \\
Domain & $y_{s}$ & 0.5 \\
geometry & $y_{\max }$ & 1 \\
Fig. 1.1 & $x_{s}$ & 0.5 \\
\hline & $D_{c}$ & 1 \\
Global & $U_{\max }$ & 0.15 \\
control & $U_{\min }$ & 0.075 \\
eq. (B.6) & $\bar{m}$ & {$\left[\bar{m}_{\min }, \bar{m}_{\max }\right]$} \\
& $c$ & {$\left[c_{\min }, c_{\max }\right]$} \\
\hline Apoptosis & $\delta$ & 1 \\
sink & $\bar{y}$ & {$\left[\lambda_{\min }, \lambda_{\max }\right]$} \\
term & $y_{s}^{-}$ & 0.02 \\
eq. (B.5) & $y_{s}^{+}$ & 0.45 \\
\hline \multicolumn{2}{|c}{} \\
\hline
\end{tabular}

\begin{tabular}{|l|c|c|}
\hline \multicolumn{3}{|c|}{ Local parameters } \\
\hline Model & Name & Value \\
\hline Initial & $M_{0}$ & {$\left[M_{0 \text { min }}, M_{0 \max }\right]$} \\
condition & $\mu_{1}$ & 0.05 \\
eq. $(2.7),(2.10)$ & $\mu_{2}$ & 0.4 \\
\hline Aging & $\gamma_{1}$ & {$\left[0, \gamma_{1 \max }\right]$} \\
velocity eq. (B.1) & $\gamma_{2}$ & {$\left[\gamma_{2 \min }, g_{\max }\right]$} \\
\hline & $c_{1}$ & {$\left[0, c_{1 \max }\right]$} \\
Maturation & $c_{2}$ & {$\left[c_{2 \min }, c_{2 \max }\right]$} \\
velocity & $\bar{u}$ & {$\left[\bar{u}_{\min }, \bar{u}_{\max }\right]$} \\
eq. (B.2) & $\tau_{h}$ & {$\left[\tau_{h \min }, \tau_{h \max }\right]$} \\
\hline Mesoscopic & $b_{1}$ & {$\left[b_{1 \min }, b_{1 \max }\right]$} \\
open-loop control & $\beta_{2}$ & {$\left[\beta_{2 \min }, \beta_{2 \max }\right]$} \\
eq. (3.2) & $T_{s 1}$ & \\
\hline Mesoscopic & $T_{s 2}$ & {$\left[b_{1 \min }, b_{1 \max }\right]$} \\
closed-loop control & $b_{1}$ & {$\left[b_{2 \min }, b_{2 \max }\right]$} \\
eq. (B.7) & $b_{2}$ & {$\left[b_{3 \min }, b_{3 \max }\right]$} \\
\hline
\end{tabular}

TABle C.1

List of the model parameters. The global parameters are shared by all follicles in a cohort while the local parameters are specific to individual follicles. Some parameters (highlighted in magenta) are given nominal values in all cases, while others are constrained to either explicit or implicit (highlighted in green) bounding conditions and may vary from one simulation or one follicle to another. Amongst them, the parameters ruling the local control variable correspond either to the open-loop (highlighted in cyan) or to the closed-loop (highlighted in blue) situation.

C.3.1. Identification of a single ovulatory trajectory. In this paragraph, we make use of the piecewise constant, open-loop approximation of the nonlocal control $u_{f}^{o l}(t)$, introduced in Eq. (3.2), and of the results obtained in section 3.3 to derive an initial guess for a parameter set of an ovulatory trajectory.

We first begin by assessing Eq. 3.5 with the specific formulation of $g_{f}$ in (B.1) and the factor expression of $h_{f}$ obtained from (B.2) and (B.3)

$$
\begin{aligned}
h_{f}(y, u) & =-\tau_{h}\left(y-r_{1}(u)\right)\left(y-r_{2}(u)\right), \text { with } r_{1}(u)<0<y_{s}<r_{2}(u), \\
T_{h_{f}}\left(y_{0}, y_{1}\right) & =\frac{1}{\tau_{h}\left(r_{2}(u)-r_{1}(u)\right)} \ln \left(\frac{y_{1}-r_{1}(u)}{y_{1}-r_{2}(u)} \frac{y_{0}-r_{2}(u)}{y_{0}-r_{1}(u)}\right) .
\end{aligned}
$$

Inverting Eq. (C.1) and substituting either $T_{h}=T_{G 1}$ or $T_{h}=T-T_{e}$ depending on whether $y_{1} \leq y_{s}$ or not, we get

$$
\begin{array}{ll}
y_{1}\left(T_{G 1}, y_{0}\right)=\frac{r_{1}-r_{2} R}{1-R} \quad & \text { with } R=\exp \left(T_{G 1} \tau_{h}\left(r_{2}-r_{1}\right)\right) \frac{y_{0}-r_{1}}{y_{0}-r_{2}}, \\
& \text { or } R=\exp \left(\left(T-T_{e}\right) \tau_{h}\left(r_{2}-r_{1}\right)\right) \frac{y_{s}-r_{1}}{y_{s}-r_{2}}, \quad x_{1}=x_{s}+T .
\end{array}
$$

Times $T_{P+A}, T_{D+A}$ and $T_{e}$ can be roughly associated with crucial steps in follicular development, respectively: the transition from a FSH-responsive to a FSH-dependent state, the transition towards dominancy (follicle selected for ovulation), and the exhaustion of the proliferative resources of the follicle at the time of the latest cell cycle exits. We then follow a simple strategy that consists in taking as a priori observations 
the first switching time $T_{s 1}^{o b s}$, that will be made coincident with an onset time of apoptosis $\left(T_{P+A}\right)$, the exit time $T_{e}^{o b s}$ and the corresponding cell numbers and estimating the values of the parameters in the $h_{f}, g_{f}$ and $u_{f}^{o l}$ functions from a multi-objective optimization step using the stochastic algorithm CMAES [8]. The set of (constrained) parameters to be optimized is

$$
\left\{p=\left(\gamma_{1}, \gamma_{2}, c_{1}, c_{2}, \tau_{h}, \bar{u}, b_{1}, \beta_{2}, T_{s_{2}}\right)\right\}
$$

while the criterion reads

$$
\begin{array}{cl}
J_{o l}(p)=\sum_{i=1}^{4} \alpha_{i} f_{i}(p), & p^{\star}=\underset{p}{\operatorname{argmin}}\left(J_{o l}(p)\right) \\
\text { with } f_{1}(p)=\left|T_{s 1}-T_{s 1}^{o b s}\right|^{2}, & f_{2}(p)=\left|T_{e}-T_{e}^{o b s}\right|^{2}, \\
f_{3}(p)=\left|m_{o v}^{0}\left(T_{s 1}\right)-m_{s 1}^{o b s}\right|^{2}, & f_{4}(p)=\mid m_{o v}^{0}\left(T_{e)}-\left.m_{e}{ }^{o b s}\right|^{2} .\right.
\end{array}
$$

Weights $\alpha_{i}, i=1, \ldots, 4$ are fitted numerically. An initial guess for $T_{s 1}$ can be assessed from the time corresponding to the diameter value $(2.5 \mathrm{~mm})$ coinciding with the onset of FSH-dependency (delimited by green dashed lines on Figure C.1): $T_{s 1}{ }^{o b s} \approx 4.0$. We then identify this value with that of $T_{P+A}(\mu)$ assessed at the mean initial maturity $\mu=\left(\mu_{1}+\mu_{2}\right) / 2$, using formulas (C.1) and (3.15). Similarly, the initial guess for the exit time $T_{e}$ is assessed from $T_{e}^{o b s}=9$ (delimited by black dashed lines on Figure C.1), and this value is matched with the computation of the exit time assessed at the lowest maturity $\mu=\mu_{1}$ in formula (3.16). In the open-loop framework, the observed cell numbers $m_{o v}^{0}\left(T_{s 1}\right) \approx 3.16$ and $m_{o v}^{0}\left(T_{e}\right) \approx 6.7$ can be in addition matched with their rough theoretical counterparts derived from the simplifying expression (3.17). This is of course a crude estimation of the evolution in time of the cell number, since it amounts to dealing with the population as if it was fully proliferating and with a point initial distribution in maturity, but it leads nevertheless to quite satisfactory results for a first trial. It might also be worth noting that, at the end of this step, the estimated values $T_{s 1}^{\star}=4.3, T_{s 2}^{\star}=6.0$ and $T_{e}=8.0$ differ from their initial guesses which were set respectively to $T_{s 1}^{o b s}=4.0, T_{s 2}^{o b s}=5.14$ and $T_{e}^{o b s}=9.0$ using the data set in Figure C.1.

Starting from $p^{\star}$ as an initial guess (see Table C.2), we next proceed to the direct comparison of the simulated outputs, obtained by simulating the 2D PDE model in the open-loop framework, with the observed cell numbers, and perform a classical, CMAES-based optimization with a single objective criterion:

$$
J_{c}(p ; q)=\sum_{i=1}^{n}\left|m_{o v}^{0}\left(t_{i}\right)-m^{o b s}\left(t_{i}\right)\right|^{2}, \quad \hat{p}=\underset{p}{\operatorname{argmin}}\left(J_{c}(p ; q)\right)
$$

where $t_{i}$ corresponds to the sampling times and $m^{o b s}\left(t_{i}\right)$ to the observed cell number at each time (whole set of red crosses on Figure C.1), with the set of estimated parameters $p=\left(\gamma_{1}, \gamma_{2}, \tau_{h}\right)$, where $\gamma_{1}$ and $\gamma_{2}$ belong to $\Omega_{g}$ while the other parameters are kept equal to their values in $p^{\star}: q=\left(c_{1}^{\star}, c_{2}^{\star}, \bar{u}^{\star}, b_{1}^{\star}, \beta_{2}^{\star}, T_{s_{1}}^{\star}, T_{s_{2}}^{\star}\right)$.

Now, going back to the full, closed-loop formulation of the model, we can draw some equivalence with the open-loop case to fix starting values for the parameters of the $S(M)$ (eq. B.6) and $b_{f}$ (eq. B.7) functions. We thus deduce the starting value of $b_{3}=7.5$ from the ovarian maturity reached at $T_{s 1}=4.3$ (green dashed lines 
on the top center panel in Figure 4.1) and that of $\bar{m}=16.0$ from the maturity at $T_{s 2}=6$ (blue dashed lines on the same panel). Keeping unchanged the values of parameters $q=\left(c_{1}^{\star}, c_{2}^{\star}, \bar{u}^{\star}, b_{1}^{\star}\right)$ in $\hat{p}$ (inherited from $\left.p^{\star}\right)$, we search for the parameters $p=\left(\gamma_{1}, \gamma_{2}, \tau_{h}, b_{2}, b_{3}, \bar{m}, c, \lambda\right)$ using an augmented version of criterion (C.5):

$$
\begin{gathered}
J_{c c}(p ; q)=J_{c}(p ; q)+C_{o v}(p ; q), \quad \tilde{p}=\underset{p}{\operatorname{argmin}}\left(J_{c c}(p ; q)\right) \\
\text { with } C_{o v}(p ; q)=\left(U\left(T_{e}\right)-U_{\min }\right)+\max \left(0, D_{o v}\left(T_{e}\right)-\alpha_{R} m_{o v}^{0}\left(T_{e}\right)\right) .
\end{gathered}
$$

The first term in the penalization criterion (C.7) ensures that the single follicle evolves in a realistic hormonal environment (including the drop in FSH levels). The second term limits the cumulative amount of cells lost by apoptosis. Indeed, even if apoptosis is a physiological process occurring also in healthy follicles, its incidence is much lower (an order of magnitude of tenfold) in ovulatory follicles than in atretic ones [10], and we can roughly consider that the cumulated cell loss does not exceed $\alpha_{R}=10 \%$ of the cell number at ovulation time.

C.3.2. Calibration of interacting ovulatory and atretic trajectories. We finally dispose of a reasonable parameter set $\tilde{p}$ (see values in Table C.2, and solid black lines in top panels of Figure 4.1) for a closed-loop ovulatory trajectory that we now intend to couple with another trajectory corresponding to an atretic follicle. Letting the local parameters of $\tilde{p}$ unchanged for the ovulatory follicle (some of them being inherited from $p^{\star}$ or $\left.\tilde{p}\right): p_{o v}=\left(\tilde{\gamma}_{1}, \tilde{\gamma}_{2}, c_{1}^{\star}, c_{2}^{\star}, \bar{u}^{\star}, \tilde{\tau}_{h}, b_{1}^{\star}, \tilde{b}_{2}, \tilde{b}_{3}\right)$, we search for a parameter set combining parameters specific to the atretic follicle with those defining the global control (common to both follicles). We expect at the same time that

1. the cell number increases significantly with respect to its initial value up to the selection time,

2. the increase in the cell number remains moderate,

3. the cell loss becomes substantial after this time, with a final cell number clearly lower than the maximal number reached along the atretic trajectory. Denoting by $m_{f}^{\star}$ the maximum cell number reached by follicle $f$ over the time window we express these requirements within a multi-criterion function

$$
C_{a t r}(p ; q)=\nu_{1}\left|\theta_{1}-m_{a t r}^{\star}\right|^{+}+\nu_{2}\left|m_{a t r}^{\star}-\theta_{2}\right|^{+}+\nu_{3} \frac{\left|m_{a t r}^{0}\left(t_{f}\right)-\theta_{3} m_{a t r}^{\star}\right|^{+}}{m_{a t r}^{\star}},
$$

where $\theta_{1}$ (respectively $\theta_{2}$ ) is the minimum (resp. maximum) cell number value below (resp. beyond) which the computed cell number contributes to the fit function, while $\theta_{3}$ measures cell loss due to atresia. Weights $\nu_{1}, \nu_{2}$ and $\nu_{3}$ are fitted numerically. At the same time, the new atretic trajectory should be still compatible with the ovulatory behavior of the other follicle, which is ensured by the constraint $C_{o v}$. In addition, we select admissible fixed values for the subset of parameters $\left(c_{1}^{a t r}, c_{2}^{a t r}, \bar{u}^{a t r}, b_{1}^{a t r}\right)$, as described in Appendix B.2. We therefore seek a minimum of

$$
J_{c o h}(p ; q)=C_{a t r}(p ; q)+C_{o v}(p ; q), \quad p_{c o h}=\underset{p}{\operatorname{argmin}}\left(J_{c o h}(p ; q)\right)
$$

with $\left\{p=\left(\gamma_{1}^{a t r}, \gamma_{2}^{a t r}, \tau_{h}^{a t r}, b_{2}^{a t r}, b_{3}^{a t r} ; \bar{m}, c, \lambda\right)\right\},\left\{q=\left(p_{o v} ; c_{1}^{a t r}, c_{2}^{a t r}, \bar{u}_{a t r}, b_{1}^{a t r}\right)\right\}$. 


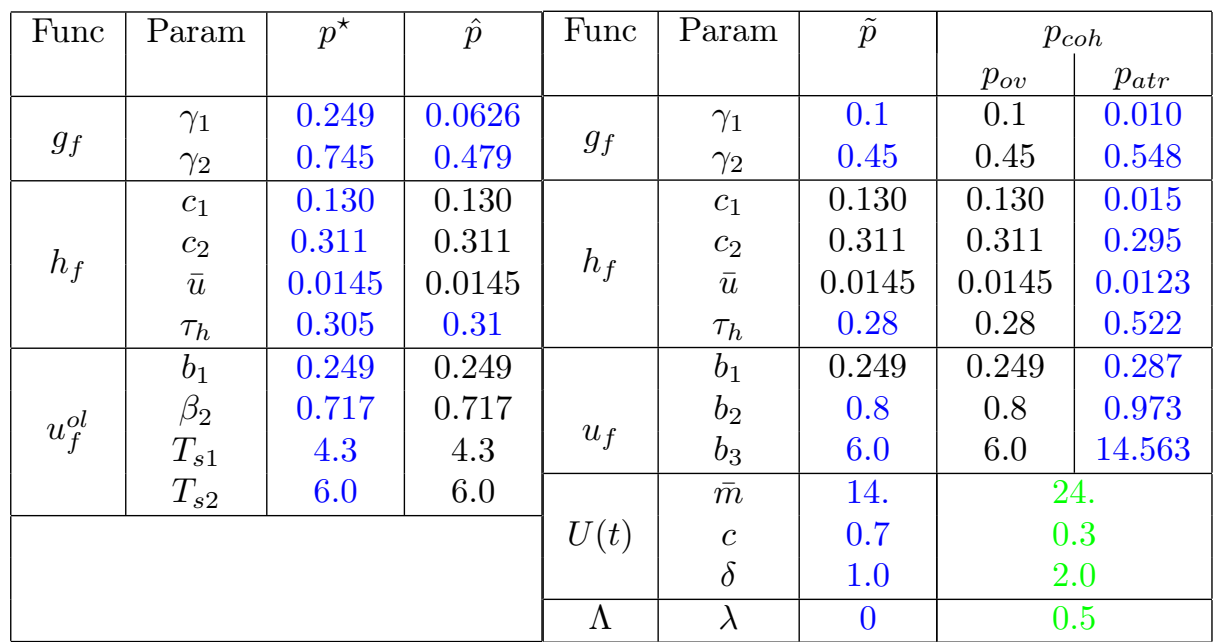

TABle C.2

Parameter values for the different parameter sets introduced in section C.3.1 and C.3.2. Parameters with fixed nominal values are $\bar{y}=0.02, M_{0}=1, g_{\min }=0.45$, and $g_{\max }=0.55$, as well as those in Table C.1. $p^{\star}$ is obtained from the particle-derived criterion $J_{o l} . \hat{p}$ is obtained from criterion (C.5) and requires the simulation of the PDE model in the open loop setup. $\hat{p}$ is used to obtain the preliminary fitted cell numbers shown on the rightmost top panel of Figure 4.1. $p_{o v}$ and $p_{a t r}$ are the final outputs of the whole estimation procedure. For $p_{\text {ov }}$, the bounds on the aging velocity parameters $\gamma_{1}$ and $\gamma_{2}$ are set using (B.14). For $p_{a t r}$, the admissible initial guess is obtained from constraint (B.19), as described in Appendix B.2. The values highlighted in color (blue or green) are the result of the current optimization set, while those left in black are inherited from the previous steps.

C.4. Flow charts for parameter identification. We now summarize the different identification steps leading to the final set of parameters $p_{\text {coh }}$ in Table C.2. We follow a sequential procedure, whose main steps are summarized in Figure C.2, and that involves the successive CMAES-based optimization of the four criteria $J_{o l}, J_{c}$, $J_{c c}, J_{a t r}$ (Step 1, 2, 3 and 5 in Figure C.3). Depending on the size of the parameter set entering the optimization procedure, the dimension of the parameter space vary between 3 (in the case of $\hat{p}$ ) and 9 (in the case of $p^{\star}$ ). Fortunately, the search for $p^{\star}$ does not require the simulation of the PDE model since it is only based on the computation of the characteristic times. Hence, although only few a priori bounds on the 9 unknown parameter values are provided in this case, the computational cost associated with the assessment of $J_{o l}$ remains largely tractable ${ }^{\ddagger}$. In contrast, Steps 2, 3 and 5 do need to perform numerical simulations of the PDEs, from the finite volume numerical method described in [3, 4], with $N_{x} \times N_{y}=40^{2}$ grid points per cycle and $\mathrm{CFL}=0.4$. Besides the optimization steps, Step 4 is needed to select admissible values for parameters of atretic follicles $\left(c_{1}^{a t r}, c_{2}^{a t r}, \bar{u}^{a t r}, b_{1}^{a t r}\right)$ as described in Appendix B.2, before the assessment of $J_{a t r}$ in Step 5 .

\footnotetext{
${ }^{\ddagger}$ If we merge weights $\left(\alpha_{i}\right)_{i=1, \ldots, 4}$ with the set of unknown parameters, the solution found by CMAES is a weak Pareto minimum favoring only the first objective (good coincidence in time between the theoretical value of $T_{s 1}$ and its observed value). In contrast, if we arbitrarily impose $\alpha_{i}=1$ for all $i=1, \ldots, 4$ the fit is much more balanced over the four objectives. Similarly we set $\nu_{1}=10, \nu_{2}=1, \nu_{3}=20, \theta_{1}=25, \theta_{2}=30$ and $\theta_{3}=0.5$ in (C.8) to obtain the parameters $p_{a t r}$.
} 


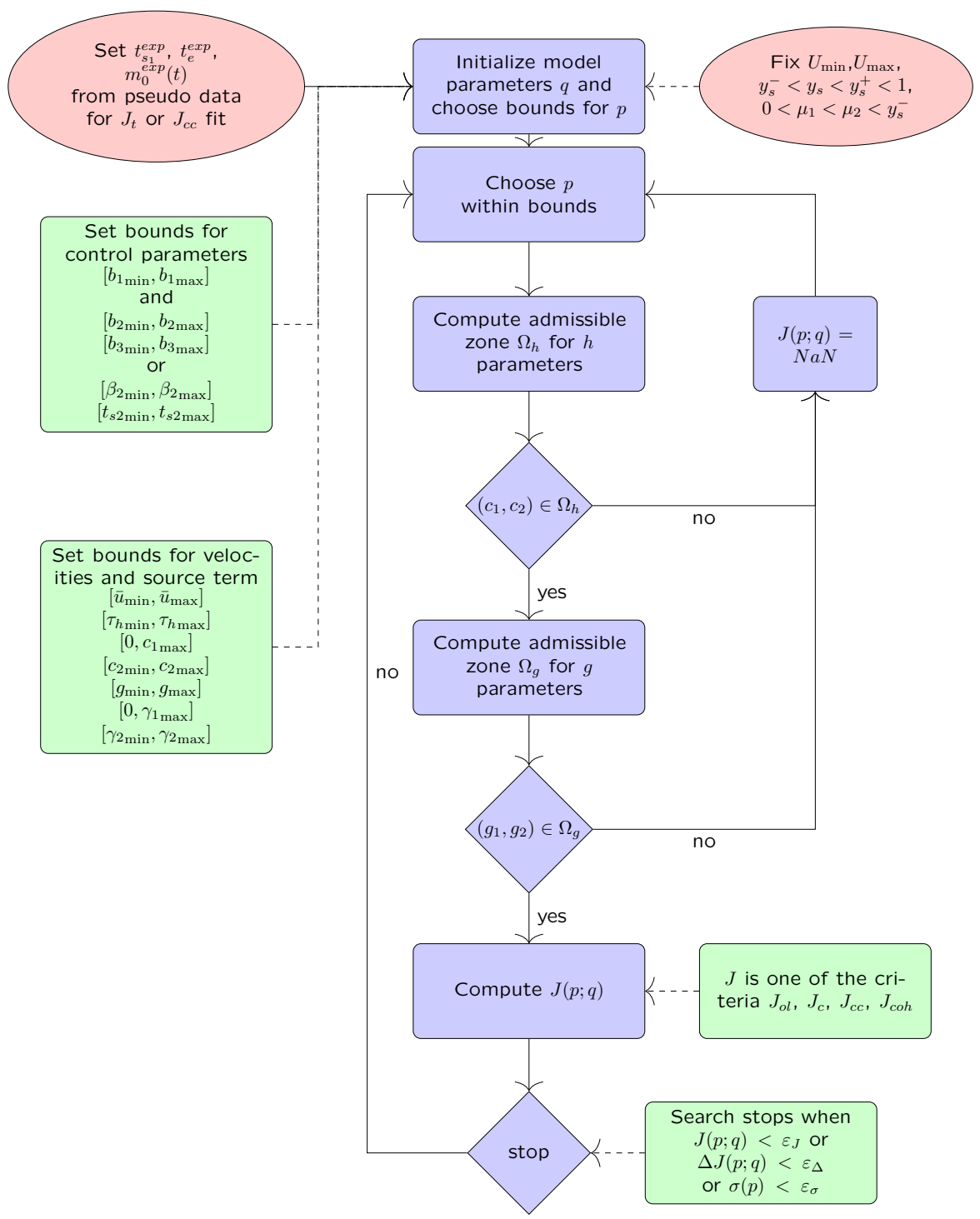

Figure C.2. Flow chart for the CMAES-based optimization of criteria (C.2,C.5, C.6,C.9). The Python library cma was used to find the parameter values corresponding to local minima of the criterion. 


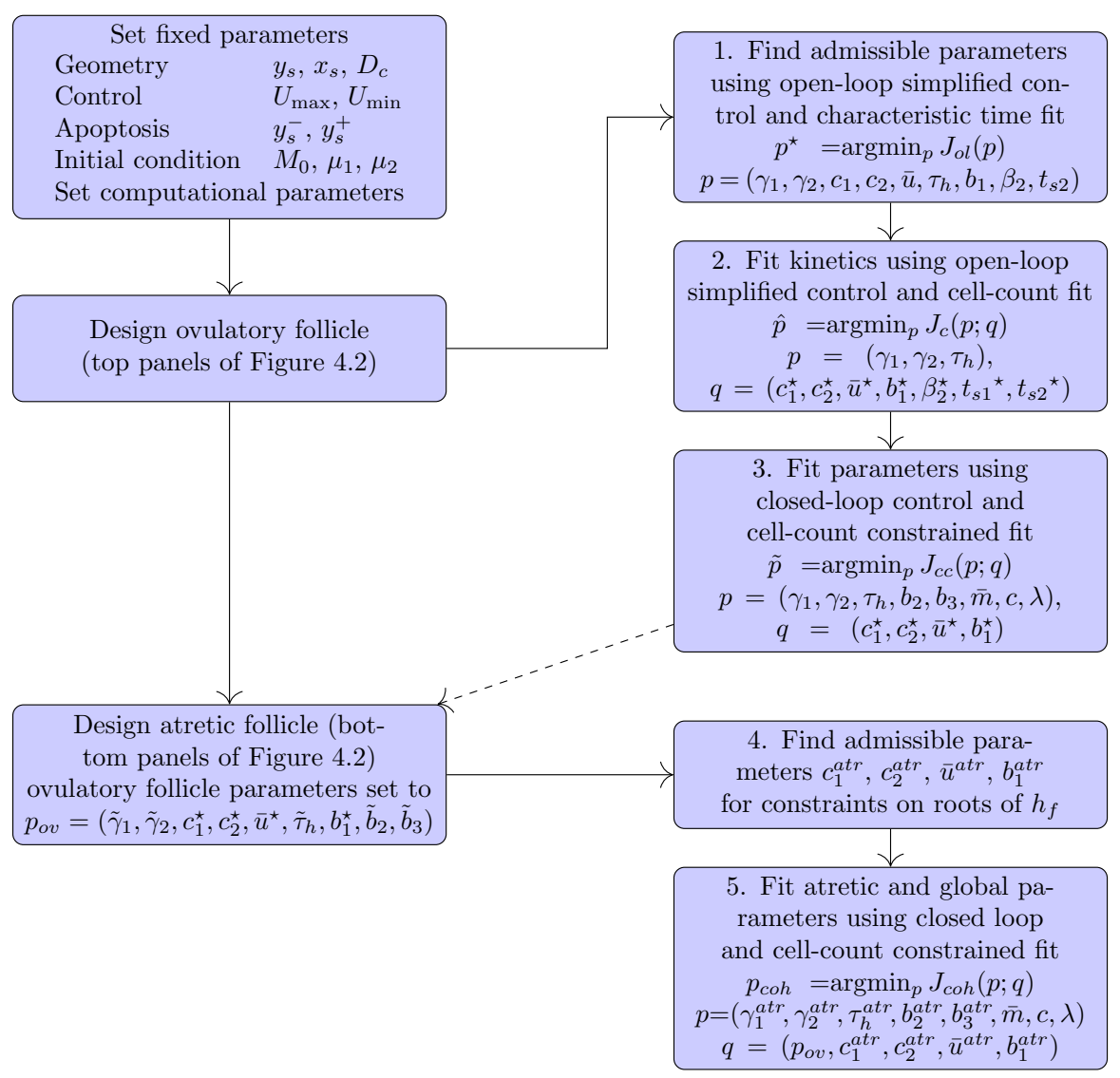

Figure C.3. Flow chart for parameter identification. In steps 2, 3 and 5, the whole parameter set is subdivided into set $q$, whose values were obtained from the previous optimization steps (respectively 1, 2 and 3) and are left unchanged, and set $p$, that is subject to the current optimization procedure. 


\section{Annex References} REFERENCES

[1] M. Adimy, F. Crauste, and S. Ruan. A mathematical study of the hematopoiesis process with applications to chronic myelogenous leukemia. SIAM J. Appl. Math., 65:1328-1352, 2005.

[2] B. Aymard, F. Clément, F. Coquel, and M. Postel. Numerical simulation of the selection process of the ovarian follicles. ESAIM Proc., 28:99-117, 2012.

[3] B. Aymard, F. Clément, F. Coquel, and M. Postel. A numerical method for transport equations with discontinuous flux functions: Application to mathematical modeling of cell dynamics. SIAM J. Sci. Comput., 35:6, 2013.

[4] B. Aymard, F. Clément, and M. Postel. Adaptive mesh refinement strategy for a non conservative transport problem. ESAIM Math. Model. Numer. Anal., 90:4, 2014.

[5] F. Clément, M.-A.Gruet, P. Monget, M. Terqui, E. Jolivet, and D. Monniaux. Growth kinetics of the granulosa cell population in ovarian follicles: an approach by mathematical modelling. Cell Prolif., 30:255-570, 1997.

[6] F. Clément, D. Monniaux, J. Stark, K. Hardy, J.-C.Thalabard, S. Franks, and D. Claude. Mathematical model of FSH-induced cAMP production in ovarian follicles. Am. J. Physiol. (Endocrinol. Metab.), 281:E35-E53, 2001.

[7] N. Echenim, D. Monniaux, M. Sorine, and F. Clément. Multi-scale modeling of the follicle selection process in the ovary. Math. Biosci., 198:57-79, 2005.

[8] N. Hansen and A. Ostermeier. Adapting arbitrary normal mutation distributions in evolution strategies: The covariance matrix adaptation. In Evolutionary Computation, 1996., Proceedings of IEEE International Conference on, pages 312-317. IEEE, 1996.

[9] J.Y. Jiang, G. Macchiarelli, B.K. Tsang, and E. Sato. Capillary angiogenesis and degeneration in bovine ovarian antral follicles. Reproduction, 125:211-223, 2003.

[10] P.D. Jolly, P.R. Smith, D.A. Heath, N.L. Hudson, S. Lun, L.A. Still, C.H. Watts, and K.P. McNatty. Morphological evidence of apoptosis and the prevalence of apoptotic versus mitotic cells in the membrana granulosa of ovarian follicles during spontaneous and induced atresia in ewes. Biol. Reprod., 56(54):837-846, 1997.

[11] E.A. McGee and A.J. Hsueh. Initial and cyclic recruitment of ovarian follicles. Endocr. Rev., 21(2):200-214, 2000.

[12] P. Michel. Multiscale modeling of follicular ovulation as a mass and maturity dynamical system. Multiscale Model. Simul., 9:282-313, 2011.

[13] D. Monniaux, C. Huet, N. Besnard, Clément F., M. Bosc, C. Pisselet, P. Monget, and J.C. Mariana. Follicular growth and ovarian dynamics in mammals. J. Reprod. Fertil. Suppl., 51(1):3-23, 1997.

[14] C. Pisselet, F. Clément, and D. Monniaux. Fraction of proliferating cells in granulosa during terminal follicular development in high and low prolific sheep breeds. Reprod. Nutr. Dev., 40:295-304, 2000.

[15] J. P. Ravindra, N. C. Rawlings, A. C. O. Evans, and G. P. Adams. Ultrasonographic study of ovarian follicular dynamics in ewes during the oestrous cycle. J. Reprod. Fertil., 101(2):501509, 1994.

[16] R.J. Scaramuzzi, D.T. Baird, B.K. Campbell, M.-A. Driancourt, J. Dupont, J.E. Fortune, R.B. Gilchrist, G.B. Martin, K.P. McNatty, A.S. McNeilly, P. Monget, Monniaux D., C. Viñoles, and R. Webb. Regulation of folliculogenesis and the determination of ovulation rate in ruminants. Reprod. Fertil. Dev., 23:444-467, 2011.

[17] P. Shang and Z. Wang. Analysis and control of a scalar conservation law modeling a highly re-entrant manufacturing system. J. Differential Equations, 2(250):949-982, 2011.

[18] B.M. Toosi, S.V. Seekallu, and N.C. Rawlings. Effects of the rate and duration of physiological increases in serum fsh concentrations on emergence of follicular waves in cyclic ewes. Biol. Reprod., 83(4):648-655, 2010.

[19] C. G. Tsonis, L. P. Cahill, R. S. Carson, and J. K. Findlay. Identification at the onset of luteolysis of follicles capable of ovulation in the ewe. J. Reprod. Fertil., 70(2):609-614, 1984.

[20] C.G. Tsonis, R.S. Carson, and J.K. Findlay. Relationships between aromatase activity, follicular fluid oestradiol-17 $\beta$ and testosterone concentrations, and diameter and atresia of individual ovine follicles. J. Reprod. Fert., 72:153-163, 1984.

[21] K.E. Turnbull, A.W. Braden, and P.E. Mattner. The pattern of follicular growth and atresia in the ovine ovary. Aust. J. Biol. Sci., 30(3):229-241, 1977.

[22] K.E. Turnbull, P.E. Mattner, J.M. George, and R.J. Scaramuzzi. The relation between patterns of ovarian follicle growth and ovulation rate in sheep. Aust. J. Biol. Sci., 31(6):649-655, 1978.

[23] J.J. Tyson and B. Novak. Temporal organization of cell cycle. Curr. Biol., 18:R759-768, 2008. 